\title{
Retos educativos ante los riesgos emergentes en el ciberespacio: claves para una adecuada prevención de la cibervictimización en menores*
}

\section{Educational challenges of emerging risks in cyberspace: foundations of an appropriate strategy for preventing online child victimisation}

Dra. Irene MONTIEL. Profesora Ayudante Doctora. Universitat Internacional de Catalunya (imontiel@uic.es).

Dr. José R. AGUSTINA. Profesor Agregado (acreditado como Catedrático). Universitat Internacional de Catalunya (jragustina@uic.es).

\section{Resumen:}

El avance tecnológico ha transformado en pocos años la forma de interactuar y comunicarse entre las personas, especialmente entre los denominados adolescentes digitales. Su impacto en las actividades cotidianas y en la cultura dominante ha propiciado, asimismo, un incremento en la exposición a riesgos psicológicos y criminológicos por parte de los menores. Ante esta nueva realidad psicosocial, se plantean nuevos retos educacionales que deben partir de un adecuado diagnóstico de la psicología y pedagogía en relación con los adolescentes.

Las teorías e investigaciones criminológicas vienen tratando de identificar los factores de riesgo y de protección con la finalidad de comprender con mayor profundidad los procesos de victimización y mejorar las estrategias de prevención. En este contexto, conviene avanzar en programas educativos que tengan

\footnotetext{
* Este trabajo ha sido realizado en el marco del proyecto de investigación «Criminología, evidencias empíricas y política criminal. Sobre la incorporación de datos científicos para la toma de decisiones en relación con la criminalización de conductas». Referencia: DER2017-86204-R, financiado por la Agencia Estatal de Investigación (AEI)/Ministerio de Ciencia, Innovación y Universidades y la Unión Europea a través del Fondo Europeo de Desarrollo Regional FEDER - «Una manera de hacer Europa».

Fecha de recepción de la versión definitiva de este artículo: 12-02-2019.

Cómo citar este artículo: Montiel, I. y Agustina, J. R. (2019). Retos educativos ante los riesgos emergentes en el ciberespacio: claves para una adecuada prevención de la cibervictimización en menores $\mid$ Educational challenges of emerging risks in cyberspace: foundations of an appropriate strategy for preventing online child victimisation. Revista Española de Pedagogía, 77 (273), 277-294. doi: https://doi.org/10.22550/REP77-2-2019-03
} 
en cuenta las carencias y vulnerabilidades personales, familiares y situacionales de los menores, a fin de fomentar el desarrollo de personas resilientes que sepan afrontar con éxito los riesgos que se fraguan y surgen en el ciberespacio. Mediante el análisis de las concretas formas de cibervictimización se pretende señalar algunas pautas basadas en la investigación criminológica y en los procesos de victimización más frecuentes en los entornos en que se desenvuelven los menores, de modo que puedan servir en el diseño de los programas educativos dirigidos a padres, educadores y potenciales víctimas.

Descriptores: cibervictimización en menores, ciberpsicología, educación en el ciberespacio, pedagogía en valores prosociales, comunicación para la prevención del delito, teorías criminológicas aplicables en educación, cyberbullying, grooming, sexting.

\section{Abstract:}

In just a few years, technological changes have transformed how people interact and communicate with each other, in particular among so-called digital adolescents. The impact of technology on routine activities and mainstream culture has led to an increase in young peoples' exposure to psychological and criminological risks. As a result of this new psychosocial trend, new educational challenges are appearing and it is becoming more necessary to react to these challenges on the basis of an adequate diagnosis of psychology and pedagogy relating to adolescents.

Criminological theories and research have tried to identify risk and protection factors to understand victimisation processes in more depth and improve prevention strategies. In this context, it is necessary to develop educational programs that consider personal, familial, and situational vulnerabilities and weaknesses in order to foster resilient individuals who can successfully confront the risks inherent to cyberspace. By analysing specific forms of cybervictimisation, guidelines based on criminological research and the most frequent victimisation processes will be identified in order to improve design of educational programs focused on parents, educators and potential victims.

Keywords: cybervictimisation of minors, cyberpsychology, education in cyberspace, pedagogy of prosocial values, communicating to prevent crime, criminological theories useful for education, cyberbullying, grooming, sexting.

\section{Antropología del smartphone y teorías criminológicas aplicadas a la cibervictimización adolescente}

Con la proliferación y extensión del uso de las TIC en la sociedad actual se ha venido a instaurar un nuevo paradigma relacional en las interacciones personales y sociales. La línea que separa el yo-real y el yo-digital cada vez es más tenue y discontinua. En efecto, en las actividades cotidianas de las personas y, de modo particular, en los denominados nativos digitales, la comunicación se apoya cada vez más en soportes virtuales. E incluso el mundo offline 
y la realidad online se llegan a confundir, por ejemplo, con la realidad aumentada. Toda esta transformación del mundo físico y su traslación al ciberespacio ha producido importantes mutaciones psicológicas, culturales y, como veremos, también victimológicas.

Como se ha señalado en otras publicaciones, ciberespacio y psicopatología son dos dimensiones que se retroalimentan y, de hecho, se llega a producir en ocasiones entre ofensores y víctimas «una cierta simbiosis psicopatológica» (Agustina, 2014; Gassó, Fernández-Cruz, Montiel, Martin-Fumadó y Agustina, 2018). "What elements of cyberspace lead to this weakening of the psychological barriers that block hidden feelings and needs?» ${ }^{1}$ - se pregunta Suler (2004, p. 322)—. Sin duda, el ser humano en la era digital presenta, junto a otras consecuencias positivas, una mayor vulnerabilidad. Más allá del transhumanismo, conviene poner el foco de atención en los cambios antropológicos que, en sí mismos, producen los cambios tecnológicos en la esfera del obrar humano en todas sus dimensiones: percepción, conocimiento, aprendizaje, comunicación, interacción y, eventualmente, victimización.

Miller (2018), al referirse al nuevo campo de estudio acuñado como antropología digital, señala que en los análisis etnográficos se debe poner mayor énfasis en aquellas formas de cultura digital que se han convertido en una realidad omnipresente, como son las redes sociales y los smartphones. Desarrollando la idea primigenia de Ortega y Gasset (1914), se puede afirmar hoy en cuanto a la identidad per- sonal que el yo soy yo y mis circunstancias está condicionado fuertemente por la dependencia del smartphone. Y si al principio se celebró la red digital como un medio de libertad ilimitada, esa ilusión ha evolucionado hasta convertirse en control y vigilancia totales, siendo así que los residentes del panóptico digital se comunican intensamente y se desnudan por su propia voluntad (Han, 2014).

Esta era de la transparencia total posee un impacto significativo en el estudio antropológico, psicológico y criminológico del ser humano. Siguiendo a Favero y Theunissen (2018), las tecnologías digitales, a modo de cuaderno de bitácora digital, están entrando en la vida de los antropólogos, en su trabajo de campo y en todo su quehacer, dando forma a cómo se registran, procesan, analizan y comunican sus hallazgos (Sanjek y Tratner, 2015). Los smartphones, de hecho, han contribuido a este giro copernicano, en tanto que se han convertido en parte integrante de nuestras actividades cotidianas (Collins et al., 2017; Lapenta, 2011; Pink y Hjorth, 2012; Tacchi, Kitner y Crawford, 2012): incluso, en la medida en que esas tecnologías se van implantando o incrustando en el propio cuerpo, estamos asistiendo a un progresivo entrecruzamiento entre cuerpos materiales y tecnologías digitales móviles (Favero, 2016; Ibrahim, 2015; Rettberg, 2014).

La anticipación en la edad de acceso a las TIC experimentada en los últimos años ha supuesto cambios muy significativos en las actividades cotidianas en el ciberespacio por parte de los menores (García, 2017). 
Estos interactúan virtualmente fuera del hogar mucho antes y en todo momento, accediendo a tales interacciones desde el smartphone, que se ha convertido no solo en una ventana al mundo, sino en un verdadero espacio ampliado de su victimización (Montiel y Agustina, 2018). Sin duda, el ciberespacio se configura como un nuevo espacio de oportunidad criminal (Miró, 2012) en el que niños, niñas y jóvenes continúan siendo protagonistas, especialmente de las nuevas formas de cibercriminalidad social (Miró, 2012), es decir, aquellos fenómenos criminales que engloban distintas formas de victimización interpersonal online como el cyberbullying, el online grooming o el sexting indeseado, de los que hablamos más adelante.

Desde las teorías criminológicas tres son los enfoques decisivos en esta era digital: la teoría del autocontrol (Gottfredson y Hirschi, 1990), la teoría de las actividades cotidianas (Cohen y Felson, 1979) y las teorías del aprendizaje (Akers, 1990, entre otros). En efecto, en primer lugar, los niveles de autocontrol en la era digital devienen un factor individual de primera magnitud, que permiten diferir gratificaciones inmediatas en un contexto tecnológico que empuja a la impulsividad. La tendencia a desarrollar comportamientos adictivos (en relación, por ejemplo, al consumo compulsivo de todo tipo de bienes y servicios, incluyendo pornografía, juegos online y otros reclamos) debilita sin duda la voluntad de la persona y su resiliencia. En segundo lugar, el entorno cotidiano importa: los factores ambientales en forma de arquitecturas digitales, mediante el diseño de espacios en la red que fomentan el anonimato y la ausencia de vigilancia, son generadores de oportunidades para la desviación que aprovechan los agresores motivados por el escaso riesgo y esfuerzo percibido en la conducta delictiva. La vulnerabilidad del ser humano depende en gran medida de la ausencia de controles (guardianes capaces en terminología de la teoría de las actividades cotidianas) y del tiempo cada vez mayor que los jóvenes pasan conectados, interactuando con conocidos y desconocidos, sin conciencia de los peligros que les acechan o con ella pero con una limitada capacidad para sopesar las consecuencias a largo plazo que estos pueden suponer. Finalmente, en tercer lugar, las teorías del aprendizaje son también explicativas de cómo aumenta la influencia de patrones de conducta y modelos inadecuados, emergiendo de este modo con inusitada fuerza disvalores y mensajes de neutralización de conductas desviadas que acaban transformando las percepciones y valores del imaginario colectivo y la motivación adolescente.

\section{Criminología cultural y adoles- centes digitales: hacia una radio- grafía de la cultura juvenil y un fortalecimiento de la resiliencia digital}

Katz ya señaló en su célebre Seductions of crime: Moral and sensual attractions in doing evil (1988) que el problema central para explicar cómo surge la decisión de cometer un delito radica en la motivación y, en concreto, en entender cómo emerge dentro del sujeto que lo comete una «distinctive sensual dynamics» (p. 4) ${ }^{2}$. La verdadera naturaleza del ser humano 
es emocional: la atención es sentimiento y la conciencia es sensual. El reto para explicar esa dinámica criminógena está en especificar los pasos del proceso dialéctico a través del cual una persona empodera al mundo para seducirlo a cometer un delito. Y parte de ese reto se descubre al reconocer las distintas secuencias en que se va fraguando ese espíritu de determinismo, secuencias que son lo suficientemente sutiles para que sus avances pasen inadvertidos (Katz, 1988).

Si la clave explicativa del delito es emocional y estética, los mensajes que deben dirigirse al homo sentimentalis para disuadirlo de la atracción delictiva deberían basarse en un lenguaje visual construido por valores prosociales estéticamente sustentados. En este sentido, desde la criminología cultural surge en tiempos recientes una orientación donde la dimensión de la imagen viene cobrando un protagonismo creciente. En efecto, como refiere Herrera (2014, p. 6) se abre paso hoy una nueva criminología visual, con un marcado cariz icónico: si la criminología estudia el delito, la criminología visual abarcaría el estudio de los modos en que lo visual interacciona con el delito, modelándose mutuamente ambas dimensiones. De este modo, su objetivo esencial es indagar sobre la «moderna estética visual de la criminalidad», en cuanto íntimamente ligada a una concreta ética cultural.

La nueva cultura adolescente es una cultura de la imagen transformada por el entorno tecnológico hasta dimensiones insospechadas, cristalizando en lapsychology of feedback, que se ha traducido en nuevas formas o derivaciones de trastornos psicológicos como un nuevo narcisismo digital o el denominado body dysmorphic disorder (Aiken, 2017). Hemos pasado de la cultura Kodak a la imagen en red y a la cultura Instagram (Gómez, 2012), y a la aparición de distintas etiquetas como selfitis o twitteritis (Balakrishnan y Griffiths, 2018; Starcevic, Billieux y Schimmenti, 2018). Los usuarios reciben inputs placenteros de aprobación social explícita y cuantificable (Sherman, Payton, Hernández, Greenfield y Dapretto, 2016; Sherman, Greenfield, Hernández, y Dapretto, 2018), cuya frecuencia y magnitud son impredecibles, como los likes, los views o los indicadores de búsqueda de Google (Loh y Kanai, 2014), lo que refuerza los comportamientos de búsqueda de recompensa y las conductas compulsivas (Knapp, 1976). Por otro lado, un volumen creciente de estudios han asociado los comportamientos adictivos relacionados con Internet con la alteración del procesamiento de la recompensa (por ejemplo, Lin, Zhou, Dong y Du, 2015; Yao et al., 2015), el procesamiento de emociones, la atención ejecutiva, la toma de decisiones, el control de impulsos (Oliva et al., 2014) y los mecanismos de autocontrol (Brand, Young y Laier, 2014; Greenfield, 2011). Nos hallamos ante una paradoja de la emancipación (Silva, 2018): todo apunta a mayores cotas de libertad y, sin embargo, la tecnología y la efervescencia sentimental han producido una atrofia alarmante de las capacidades de autocontrol.

Quizás ha llegado el momento de admitir que el concepto nativo digital está sobrevalorado, como apoyan diversos autores (por ejemplo, Kirschner y De Bru- 
yckere, 2017; L'Ecuyer, 2015; Rowlans et al., 2008). En este sentido, Rowlans et al. (2008), reconocen que aunque los jóvenes demuestran una gran familiaridad y agilidad técnica con las tecnologías, dependen demasiado de los motores de búsqueda y carecen de las competencias críticas y analíticas para poder entender el valor y la originalidad de la información en la web. En la misma línea, Carr (2011) afirma que los nativos digitales gravitan hacia un modo de procesamiento de la información superficial, caracterizado por el desplazamiento atencional rápido y la mínima reflexión.

\section{3. «De aquellos polvos, estos lo- dosı: vulnerabilidad, factores de riesgo, protección, y nuevas for- mas de victimización}

Los efectos de tres revoluciones sociales de enorme calado (la revolución sexual, la revolución digital y la revolución adolescente), junto a la pérdida del sentido de intimidad (Agustina, 2010a; Agustina y Gómez-Durán, 2016), han irrumpido de forma especialmente intensa en los menores digitales. De suerte que, habiéndose creado unas condiciones socio-culturales propicias a la sobreexposición a distintos tipos de riesgos, las nuevas formas de victimización no se han hecho esperar.

Así las cosas, las distintas formas de victimización online han ido adoptando características propias, dando lugar a nuevas formas de criminalidad social, como el cyberbullying, el sexting y el online grooming (Miró, 2012). Según estudios recientes, más del $50 \%$ de la población adolescente en España ha padecido alguna de estas formas de cibercriminalidad social (Miró y García, 2014; Montiel, Carbonell y Pereda, 2016), lo que puede afectar negativamente los procesos de desarrollo cognitivo, neurológico y socioafectivo de los más jóvenes, incrementando el riesgo de desarrollar trastornos psicopatológicos y problemas de conducta, además de incrementar su vulnerabilidad a la victimización en la edad adulta, como demuestran numerosos estudios sobre victimización infantil y polivictimización (Finkelhor, 2008; Finkelhor, Ormrod y Turner, 2007; Finkelhor, Turner, Ormrod y Hamby, 2009; Pereda, Guilera y Abad, 2014).

El ciberacoso entre menores o cyberbullying consiste en conductas agresivas repetidas en el tiempo, llevadas a cabo intencionadamente a través de dispositivos electrónicos, con la finalidad de agredir a una víctima que no puede defenderse fácilmente (Kowalski, Giumetti, Schroeder y Lattanner, 2014). Se trata de un fenómeno extendido entre nuestros jóvenes, pues investigaciones recientes muestran que la prevalencia entre los 12 y los 18 años se sitúa en torno al 30-50\% (Calvete, Orue y Gámez-Guadix, 2016). La invisibilidad y anonimato de sus actores y espectadores, y el efecto desinhibitorio que ello conlleva (Suler, 2004), la distancia entre víctima y agresor (especialmente emocional), la velocidad de difusión de los contenidos y la (casi) imposibilidad de destruirlos, la amplitud de la audiencia y su naturaleza omnipresente son algunos de los elementos que configuran este fenómeno como la versión mejorada y más dañina del bullying tradicional. El hostigamiento se puede dar simultáneamente por diferentes canales a 
los que víctima, agresor y espectadores están siempre conectados mediante diferentes dispositivos, en contextos y situaciones diversas, por lo que no hay lugares seguros, ni siquiera el propio hogar (Kowalski et al., 2014). Todo ello no solo promueve la conducta impulsiva y desinhibida de los perpetradores (cada vez más agresivos), sino que también facilita el rápido aislamiento de la víctima (cada vez más indefensa) y contribuye a la prolongación indefinida de su sufrimiento y la cronificación de la victimización (Slonje, Smith y Frisén, 2013), por la gran dificultad de escapar y borrar su huella digital (Montiel, 2016).

El online grooming constituye la conducta llevada a cabo generalmente por un adulto (u otro menor pero significativamente mayor que la víctima) mediante el uso de las TIC, con la finalidad de engañar, manipular o embaucar a un menor para un futuro contacto sexual online u offline (Gámez-Guadix, Almendros, Calvete y De Santisteban, 2018). Montiel et al. (2016), a partir de una muestra de 3897 menores españoles entre 12 y 17 años, observan una prevalencia del $17,2 \%$, siendo las chicas quienes presentan mayor tasa de victimización y aumentando hasta un $25,6 \%$ en los jóvenes de 16-17 años.

El grooming en sí mismo no implica necesariamente una actividad sexual, sino que constituye la estrategia de cortejo 0 seducción empleada por el agresor para acercarse al menor, captar su atención e interés, seducirlo, establecer con él un vínculo afectivo y reducir sus inhibiciones para incrementar las posibilidades de éxito cuando le plantee alguna solicitud sexual, igual que ocurre en el abuso sexual infantil tradicional (Montiel et al., 2016). Si bien ya en este proceso seductor es posible recurrir a elementos sexuales para reducir las inhibiciones de los menores (exhibición de imágenes sexuales de otros menores 0 de adultos, conversaciones sexuales, etc.). La dimensión tecnológica del abuso facilita ahora los procesos tanto de preparación del groomer (observación y selección de víctimas, empoderamiento y refuerzo de su interés sexual en menores, etc.), como de captación de potenciales víctimas (simultaneidad de víctimas y de escenarios), de establecimiento del vínculo, sexualización progresiva de la relación (aceleración y co-responsabilización) y de difusión 0 intercambio de las evidencias visuales del abuso (ganancias económicas o de estatus social en redes pedófilas) (Gassó, Fernández-Cruz, Montiel, Martín-Fumadó y Agustina, 2018).

El término sexting proviene de la contracción de «sex» $\mathrm{y}$ «texting», y en las distintas definiciones que se han venido utilizando se incluían el envío, la recepción 0 el reenvío o difusión, tanto de mensajes sexualmente explícitos, como de imágenes de los protagonistas en las que aparecen desnudos, semidesnudos o de forma sexualmente sugerente, realizándose dicha comunicación a través de teléfonos móviles, redes sociales o Internet (Agustina y Montiel, 2016). En España, el 33,5\% de los adolescentes practican sexting, siendo más frecuente el sexting entre los adolescentes de mayor edad (Villacampa, 2017) y alcanzando su mayor pico entre los jóvenes adultos (Gámez-Guadix, Almendros, Borrajo y Calvete, 2015). Algunas de las principales 
complejidades de este fenómeno estriban tanto en las implicaciones legales que suscita el problema, como en la variedad de contenidos, comportamientos, motivaciones y medios que comprende (Drouin, Vogel, Surbey y Stills, 2013). También en el importante desacuerdo existente sobre dónde situar la línea que separa las conductas de exploración sexual entre iguales que pueden considerarse normativas de aquellas otras que resultan abusivas e inapropiadas (Livingstone y Smith, 2014), que se dan en contextos agresivos o coercitivos 0 que implican pornografía infantil. Solo en estos casos podríamos hablar del sexting como una forma de violencia interpersonal en línea 0 un cibercrimen social, que bien podría ser, además, la antesala para otros cibercrímenes como el ciberacoso o el online grooming (Agustina y Montiel, 2016).

Aunque es importante señalar que la experiencia de estos riesgos online no siempre lleva aparejada la experimentación de un daño (Livingstone, Haddon, Görzig y Ólafsson, 2011), más de la mitad de las víctimas de cyberbullying (Tsitsika et al., 2014) y al menos una de cada tres víctimas de experiencias sexuales indeseadas online se siente afectado negativamente por estas experiencias (Ybarra, Mitchell y Korchmaros, 2011), especialmente las chicas, los niños/as más pequeños y aquellos/as con dificultades psicológicas (Livingstone y Smith, 2014; Whittle, Hamilton-Giachritsis, Beech y Collings , 2013). Según la revisión de Smith y Livingstone (2017), los factores predictores de la aparición de un daño derivado de experiencias de victimización online hacen referencia principalmente a tres grupos: factores de personalidad (búsqueda de sensaciones, baja autoestima, desvinculación moral y dificultades psicológicas), factores sociales (falta de apoyo parental y normas sociales) y factores digitales (prácticas o conductas online, habilidades digitales, vulnerabilidad a los reclamos que ofrecen determinados servicios y sitios web), y proponen que las intervenciones centren su atención en estos grupos de riesgo más vulnerables al daño psíquico, fortaleciendo su capacidad de resiliencia y estrategias de afrontamiento.

No debemos obviar que la mayoría de los adolescentes implicados en alguna de estas formas de violencia interpersonal en línea suele estarlo también en otras, dando lugar a situaciones de victimización online múltiple, en el caso de las víctimas (Montiel et al., 2016), o a patrones generales de comportamiento disfuncional online, en el caso de los agresores (Montiel y Carbonell, 2016), y que es precisamente esta acumulación de experiencias y conductas negativas lo que contribuye a un peor ajuste psicológico y emocional del menor (Pereda et al., 2014).

Entre los factores presentes en los grupos de adolescentes en riesgo, junto a ciertas características de los propios menores y del espacio virtual, ocupa una posición importante mantener una pobre relación con los padres, conflictiva, poco cohesionada 0 negligente. Desde la criminología, la perspectiva familiar es aquella concepción sostenida ampliamente según la cual una familia fuerte reduce la existencia del delito, mientras que una familia débil 
contribuye a que este aparezca en mayor medida. Sampson ha argumentado al respecto que la vida familiar (más que la pobreza en sí misma) es la principal fuerza motora en la generación 0 evitación del delito. La perspectiva familiar encaja bien con el resto de perspectivas criminológicas comunes. Un ambiente familiar favorable tiende a contrarrestar las fragilidades propias del ser humano, mantiene a los jóvenes alejados de situaciones desfavorables, los aparta de malas compañías, del estar inactivo u ocioso, de tentaciones no deseables, riesgos y provocaciones, fomentando al mismo tiempo el autocontrol cuando es necesario. Más aún, las teorías del control nos dicen que los niños temen a menudo avergonzar a sus padres al meterse en problemas.

La vida familiar sirve también para reducir el riesgo de victimización (Agustina, 2010b). En este sentido, numerosos estudios sitúan las variables familiares como la falta de cohesión y la conflictividad como predictores de la cibervictimización. Por ejemplo, respecto al online grooming, Whittle et al., (2013), destacan que los jóvenes que están marginados de su familia, en conflicto con sus padres o presentan dificultades familiares son vulnerables a los acercamientos sexuales online (Mitchell, Finkelhor y Wolak, 2001, 2007; Wells y Mitchell, 2008; Wolak, Finkelhor y Mitchell, 2004). A partir de estudios realizados con online groomers, se ha observado que estos reconocen a los jóvenes que buscan atención, empatía o retroalimentación por parte de personas adultas y se aprovechan de estas carencias afectivas (Webster et al., 2012; Santisteban y Gámez-Guadix, 2017).
En relación al cyberbullying, el apoyo parental aparece como un importante factor protector (Wang, Iannotti y Nansel, 2010), mientras que una pobre relación paterno/materno filial predice el acoso online (Ybarra y Mitchell, 2004). Por su parte, según Baumgartner, Sumter, Peter y Valkenburg (2012), los adolescentes que se implican en comportamientos sexuales de riesgo online como el sexting, están menos satisfechos con sus vidas, presentan niveles más altos de búsqueda de sensaciones, provienen de familias menos cohesionadas y con un menor nivel de educación, y utilizan más Internet para comunicarse, apoyando la idea de que los adolescentes que tienen problemas en su vida cotidiana pueden recurrir a Internet para suplir la pérdida de gratificación fuera de línea (Wolak, Mitchell y Finkelhor, 2003).

\section{Cultura del control versus cul- tura de la educación. La recupe- ración del concepto de virtud a través del paradigma del auto- control}

Vivimos en una sociedad obsesionada con el control y la tolerancia cero frente a cualquier tipo de riesgo, instalada en un paradigma de prevención que no ha entendido de forma adecuada que, en realidad, la educación de la propia persona y el aprendizaje progresivo en el ejercicio de una libertad responsable son mucho más determinantes. Ni el Estado, ni la modificación de las estructuras sociales y culturales que incentivan a las personas a llevar estilos de vida en los que no se valoran suficientemente los riesgos de victimización, pueden garantizar la preservación de 
los jóvenes frente a los peligros de la era digital. Los mecanismos de control externos no permean el interior del individuo $y$, aunque el ambiente es determinante a la hora de configurar el espacio de oportunidad delictiva, conviene invertir más en formar personas resilientes sin descuidar el enfoque situacional, ni la modificación de las estructuras socio-culturales ya referidas.

En este contexto, parece decisivo comprender la gradualidad necesaria en que debe desarrollarse la pedagogía de la libertad. Prevenir es sobre todo educar. Y educar en la libertad debería conllevar ofrecer motivaciones adecuadas para que los jóvenes vean los aspectos positivos y negativos de las TIC y decidan decantarse por un uso responsable de los medios tecnológicos. En todo caso, para educar hay que supervisar de forma conveniente (Osgood y Anderson, 2004). Existen muchas formas de supervisar: no se trata de un mero acompañar, aunque sin duda la sola presencia de padres y formadores puede favorecer dinámicas de conducta menos irreflexivas, más prosociales. Algunos estudios analizan la eficacia de distintas estrategias de mediación parental en el uso que hacen los menores de Internet y concluyen que no existe una relación simple y directa entre esta y las experiencias online de los menores, por lo que el mero hecho de incrementar la mediación no reduce la exposición a los riesgos online (Livingstone y Helsper, 2008) y el control parental percibido por los jóvenes solo reduce mínimamente sus comportamientos de riesgo online (Valcke, De Wever, Van Keer y Schellens, 2011).
Hay autores que sostienen que el control parental no influye en la victimización por cyberbullying (Marcum, Higgins y Ricketts, 2010; Moore, Guntupalli y Lee, 2010). Otros observan, en cambio, que algunas estrategias concretas de supervisión parental pueden ser factores protectores, como supervisar el tiempo de uso de Internet y la facilitación de información personal (Ortega-Barón, Buelga y Cava, 2016; Walrave y Heirman, 2011), mientras otras como supervisar las páginas que visitan online, usar software de filtrado o la localización física del ordenador, no funcionan (Lee y Chae, 2007; Mesch, 2009; Navarro, Serna, Martínez y Ruiz-Oliva, 2012). El impacto de la regulación parental depende, entre otras cosas, del tipo de uso que los menores hacen de Internet (Eynon y Malmberg, 2011) y del nivel de integración de esta tecnología en la vida de los jóvenes (Steeves y Webster, 2008). En el primer caso, por ejemplo, el efecto de la supervisión parental es mayor sobre los usuarios activos (todo tipo de usos, especialmente social) que sobre los usuarios normativos (comunicación, entretenimiento y búsqueda de información); y sobre los usuarios periféricos (uso limitado de Internet) que sobre cualquier otro tipo de usuario (Eynon y Malmberg, 2011).

Junto a la presencia de capable guardians $^{3}$ (Hollis, Felson y Welsh, 2013) en el entorno de las actividades cotidianas de los menores, es preciso construir un lenguaje comunicativo que sea eficaz en el contexto de los programas e instrumentos de prevención. Y ese lenguaje transmisor de valores prosociales, también en el ciberespacio, debe emitirse desde la familia como núcleo 
de socialización primordial del individuo y desde otras instancias que coadyuvan a formar la cultura del grupo o la cultura social. Los modelos que verdaderamente arrastran en la cultura adolescente tienen un peso fundamental en la conformación de ese conjunto de mensajes favorables 0 desfavorables a prácticas de riesgo.

La crisis de valores actual en que nos hallamos (incivismo libertario, desapego 0 insensibilidad moral, elevados índices de violencia interpersonal, tendencia a un egoísmo individualista atomizante, ausencia de ética en los negocios, entre otros síntomas) responde a dos problemas que se retroalimentan: un oscurecimiento de la inteligencia para conocer el bien y un debilitamiento de la voluntad para decidirse a practicarlo. Frente al primer problema, debería proponerse un retorno al concepto de virtud y al reto consistente en hacerla atractiva en el discurso educativo, político y social; y, frente al segundo, deberían plantearse fórmulas para un refortalecimiento de la estructura familiar como entorno propicio para el desarrollo de la capacidad de resiliencia y el autocontrol. Desde algunas corrientes de pensamiento dominantes se ha puesto el énfasis en un concepto de libertad sin límites (más allá de unos mínimos que, a la vista de la situación actual, parecen insuficientes). En este sentido, se ha optado por un modelo pedagógico basado en un cierto emotivismo ético que se justifica a sí mismo. En ese contexto, se debería volver a un planteamiento positivo de qué significa educar en el que se proponga de forma decidida volver a resaltar la importancia del autocontrol como contrapeso al dejarse llevar sin más por un flujo de emociones, impulsos y objetivos gratificantes a corto plazo. El constructo autocontrol no es sino la traducción contemporánea del concepto aristotélico de virtud.

Desde este punto de vista, vivimos en una sociedad anómica en la que las normas cumplen solo una función externa, de amenaza o coacción psicológica en caso de desviación. La falta de referencias objetivas básicas en el obrar moral y en el discurso político y social dominante ponen de manifiesto la magnitud de la crisis de valores que padecemos. Entre los signos de desazón del hombre posmoderno sobresale un cierto pesimismo existencial, fruto de un relativismo valorativo que, prima facie, nos iba a traer la libertad y la tolerancia. La sensación de angustia y ansiedad es ciertamente una situación paradójica en la época de la historia de la humanidad de mayor bienestar y seguridad. El modelo de felicidad basado en un hedonismo compulsivo no hace más que aumentar el vacío existencial y la falta de sentido de una sociedad que camina, más que nunca, a oscuras. Con todo, a partir de una radiografía realista, sin eufemismos, se debe tratar de construir un escenario esperanzador que devuelva a la familia, a las instancias educativas, a los medios de comunicación social y al liderazgo político ese deseo imprescindible por mejorar de forma efectiva las condiciones de desarrollo para generar ciudadanos responsables.

Partiendo de tales premisas, deberían orientarse los programas de formación de padres y educadores hacia una nueva cultura en el uso de las TIC que abogue por 
una apuesta decidida por el autocontrol como contrapunto a los efectos desinhibitorios descritos por Suler (2004), la empatía y las normas de educación en el ciberespacio (netiquette), la moderación en los hábitos de uso, la recuperación del sentido de intimidad, la conciencia de los efectos negativos de la autorreferencialidad narcisista 0 la conciencia de las consecuencias y el rastro indeleble de la propia conducta en Internet (cultura del día después). En un nivel más específico, la prevención del $c y$ berbullying, por ejemplo, puede orientarse al entrenamiento general en empatía, la modificación de creencias que apoyan la agresión, y el establecimiento de pautas más específicas para el comportamiento en Internet, incluyendo acciones que los jóvenes pueden realizar como denunciar los abusos y recoger evidencias (Smith y Livingstone, 2017). La prevención de las conductas de sexting puede centrarse en la educación sexoafectiva y la autorregulación emocional, el respeto por la intimidad propia y ajena, así como en formación sobre la arquitectura digital y los riesgos para la privacidad online que explican la pérdida de control sobre todo aquello que se publica en la red. Por su parte, la prevención de las situaciones de online grooming puede apoyarse principalmente en el refuerzo del apoyo parental y la cohesión familiar, la promoción de las relaciones afectivas saludables no coercitivas 0 asimétricas y el desarrollo de un pensamiento crítico hacia la hipersexualización de la infancia, desarticulando todas aquellas creencias erróneas y falacias que facilitan que los jóvenes se vean involucrados en relaciones abusivas online (Montiel, Carbonell y Salom, 2014).
Es importante señalar que en el ciberespacio, la dualidad víctima-agresor es cada vez menos rígida, pudiendo hablar incluso de un continuo de implicación en el que es habitual la figura de la víctima-agresiva o el agresor-victimizado (Montiel, 2016; Walrave y Heirman, 2011), existiendo suficiente evidencia empírica que avala el importante solapamiento de roles, tanto online como offline (Kowalski et al., 2014; Smith, 2014). En el mismo sentido, sabemos que los niños y niñas que son vulnerables offline, también lo son online, del mismo modo que aquellos/as que asumen riesgos en un ámbito, es más probable que los asuman también en el otro (Livingstone y Smith, 2014). En este sentido, desde el enfoque de la victimología del desarrollo, Pereda et al., (2014), observan una elevada asociación entre la condición de polivíctima y las victimizaciones por parte de cuidadores (de tipo físico y psicológico), la victimización sexual (especialmente por adultos desconocidos) y la victimización por Internet (ciberacoso no sexual y solicitudes sexuales indeseadas).

Todo ello apunta la necesidad de adoptar enfoques ecológicos e integradores que, en lugar de centrarse en la prevención de conductas concretas como el ciberacoso o el online grooming, o en roles específicos (víctima 0 agresor) 0 niveles de análisis microsistémicos (escuela o familia), asuman una visión más amplia de la victimización infantojuvenil online, en la que para muchos niños y niñas, la violencia digital puede ser una condición vital prácticamente crónica más que una experiencia puntual, lo que apunta hacia la presencia de una fuerte raíz estructural y señala la existencia de un grave problema endémico de la sociedad actual. 
En general, es necesario ajustar los adecuados mensajes de prevención a los adolescentes, elevar el grado de advertencia en cuanto a los riesgos y motivarlos para que reconsideren entrar en contacto con personas desconocidas online para paliar sus fragilidades 0 carencias psicológicas, como la soledad o la depresión (Wolak et al., 2004), así como permitirles desarrollar su capacidad de autorregulación y autocontrol para no sucumbir a la desinhibición, la pérdida de control y de libertad online, al tiempo que se potencian los factores protectores que minimizan el impacto de los riesgos. Pero para ello, es necesario ofrecerles espacios alternativos de desarrollo más atractivos y emocionantes que las pantallas, más auténticos.

Para prevenir la cibervictimización infanto-juvenil no basta con educar en competencias tecnológicas, pues como afirma L'Ecuyer (2018), la verdadera preparación para un buen uso de las tecnologías reside en la comprensión del contexto, que no se desarrolla en un entorno descontextualizado como es el contexto online, sino que se adquiere en el entorno offline, que es el mundo real. Tampoco se puede pretender que las propias aplicaciones tecnológicas ofrezcan la solución al problema, puesto que este va más allá de la arquitectura digital del ciberespacio y podemos garantizar que lo que pone en riesgo a los menores no son los ordenadores o la tecnología per se, sino las personas (Martellozzo, 2013) y consideramos que, como apunta Steve Jobs, "lo que no funciona con la educación, no se arregla con la tecnología» (Wolf, 1996).

\section{Notas}

${ }^{1}$ "¿Qué elementos del ciberespacio conducen a ese debilitamiento de las barreras psicológicas que blo. quean los sentimientos y necesidades escondidas?» (traducción propia).

2 Una «dinámica sensual distintiva» (traducción propia).

${ }^{3}$ «Guardianes capaces» (traducción propia).

\section{Referencias bibliográficas}

Agustina, J. R. (2010a). iMenores infractores 0 víctimas de pornografía infantil? Respuestas legales e hipótesis criminológicas ante el Sexting. Revista Electrónica de Ciencia Penal y Criminología, 12 (11), 1-44.

Agustina, J. R. (2010b). Conceptos clave, fenomenología, factores y estrategias en el marco de la violencia intrafamiliar. En J. R. Agustina (Ed.), Violencia intrafamiliar. Raíces, factores y formas de la violencia en el hogar (pp. 61-132). Madrid: BdeF-Edisofer.

Agustina, J. R. (2014). Cibercriminalidad y perspectiva victimológica: un enfoque general explicativo de la cibervictimización. Cuadernos de Política Criminal, 114 (3), 143-178.

Agustina, J. R. y Gómez-Durán, E. L. (2016). Factores de riesgo asociados al sexting como umbral de diversas formas de victimización. Estudio de factores correlacionados con el sexting en una muestra universitaria. Revista de Internet, Derecho y Política, 22, 21-47.

Agustina, J. R. y Montiel, I. (2017). Sexting en adolescentes: nuevos retos médico-legales. Revista Española de Medicina Legal, 43 (1), 43-44.

Aiken, M. (2017). The Cyber Effect: An expert in cyberpsychology explains how technology is shaping our children, our behavior, and our value and what we can do about it. Nueva York: Spiegel \& Grau.

Akers, R. L. (1990). Rational choice, deterrence and social learning theory in criminology: The path not taken. Journal of Criminal Law \& Criminology, 81, 653-676.

Balakrishnan, J. y Griffiths, M. D. (2018). An Exploratory Study of «Selfitis» and the Development of the Selfitis Behavior Scale. International Journal of Mental Health and Addiction, 16 (3), 722-736. 
Baumgartner, S. E., Sumter, S. R., Peter, J. y Valkenburg, P. M. (2012). Identifying Teens at Risk: Developmental Pathways of Online and Offline Sexual Risk Behavior. Pediatrics, 130 (6), 1489-1496. doi: https://doi.org/10.1542/ peds.2012-0842

Brand, M., Young, K. S. y Laier, C. (2014). Prefrontal control and Internet addiction: a theoretical model and review of neuropsychological and neuroimaging findings. Frontiers in human neuroscience, 8, 1-13. doi: https://doi. org/10.3389/fnhum.2014.00375

Calvete, E., Orue, I. y Gámez-Guadix, M. (2016). Cyberbullying Victimization and Depression in Adolescents: The Mediating Role of Body Image and Cognitive Schemas in a One-year Prospective Study. European Journal on Criminal Policy and Research, 22 (2), 271-284.

Carr, N. (2011). The shallows: what the Internet is doing to our brains. Nueva York: WW Norton.

Cohen, L. E. y Felson, M. (1979). Social Change and Crime Rate Trends: A Routine Activity Approach. American Sociological Review, 44 (4), 588-608.

Collins, S. G., Durington. M., Favero, P., Harper, K., Kenner, A. y O’Donnell, C. (2017). Ethnographic Apps/Apps as Ethnography. Anthropology Now, 9 (1), 102-18.

De Santisteban, P. y Gámez-Guadix, M. (2017). Estrategias de persuasión en grooming online de menores: Un análisis cualitativo con agresores en prisión. Psychosocial Intervention, 26 (3), 139-146.

Drouin, M., Vogel, K. N., Surbey, A. y Stills, J. R. (2013). Let's talk about sexting, baby: Computer mediated behaviors among young adults. Computers in Human Behavior, 29, A25-A26.

Eynon, R. y Malmberg, L. E. (2011). A typology of young people's Internet use: implications for education. Computers and Education, 56 (3), 585-595.

Favero, P. (2016). Analogization: Reflections on Life-Logging Cameras, Action Cams and Images' Changing Meaning in a Digital Landscape. En E. G. Cruz, E. Lehmuskallio y A. Lehmuskallio (Eds.), Digital Photography and Everyday Life: Empirical Studies on Material Visual Practices (pp. 209-27). New York: Routledge.
Favero, P. S. y Theunissen, E. (2018). With the Smartphone as Field Assistant: Designing, Making, and Testing EthnoAlly, a Multimodal Tool for Conducting Serendipitous Ethnography in a Multisensory World. American Anthropologist, 120 (1), 163-167.

Finkelhor, D. (2008). Childhood Victimization. Violence, Crime, and Abuse in the Lives of Young People. Oxford, USA: Oxford University Press.

Finkelhor, D., Ormrod, R. K. y Turner, H. (2007). Revictimization patterns in a national longitudinal sample of children and youth. Child Abuse \& Neglect, 31, 479-502.

Finkelhor, D., Turner, H., Ormrod, R. y Hamby, S. (2009). Violence, Abuse, and Crime Exposure in a National Sample of Children and Youth. Pediatrics, 124 (5), 1411-1423.

Gámez-Guadix, M., Almendros, C., Borrajo, E. y Calvete, E. (2015). Prevalence and association of sexting and online sexual victimization among Spanish adults. Sexuality Research and Social Policy, 12 (2), 145-154.

Gámez-Guadix, M., Almendros, C., Calvete, E. y de Santisteban, P. (2018). Persuasion strategies and sexual solicitations and interactions in online sexual grooming of adolescents: Modeling direct and indirect pathways. Journal of Adolescence, 63, 11-18.

García Guilabert, N. (2017). El ciberacoso. Análisis de la victimización en menores en el ciberespacio desde la teoría de las actividades cotidianas. Madrid: Editorial B de F.

Gassó, A. M., Fernández-Cruz, V., Montiel, I., Martin-Fumadó, C. y Agustina, J. R. (2018). Retos forenses ante la cibercriminalidad social en menores. Revista Española de Medicina Legal. doi: https://doi.org/10.1016/j.reml.2018.11.003

Gómez Cruz, E. (2012). De la cultura Kodak a la imagen en red: una etnografía sobre fotografía digital, 23. Barcelona: Editorial UOC.

Gottfredson, M. R. y Hirschi, T. (1990). A general theory of crime. Palo Alto, CA: Stanford University Press.

Greenfield, D. (2011). The addictive properties of Internet usage. En K. S. Young, C. N. de Arbeu (Eds.), Internet addiction: a handbook and guide to evaluation and treatment (pp. 135-53). Hoboken, NJ: John Wiley. 
Han, B. C. (2014). Psicopolitica. Barcelona: Herder. Herrera Moreno, M. (2014). Construcción cultural y prevención criminal publicista: Una revisión de casos conflictivos. Revista Electrónica de Ciencia Penal y Criminología, 16-10, 10:1-10:48.

Hollis, M. E., Felson, M. y Welsh, B. C. (2013). The capable guardian in routine activities theory: A theoretical and conceptual reappraisal. Crime Prevention and Community Safety, 15 (1), 65-79.

Ibrahim, Y. (2015). Instagramming Life: Banal Imaging and the Poetics of the Everyday. Journal of Media Practice, 16 (1), 42-54.

Katz, J. (1988). Seductions of crime: Moral and sensual attractions in doing evil. New York: Basic Books.

Kirschner, P. y de Bruyckere, P. (2017). The myths of the digital native and the multitasker. Teaching and Teacher Education, 67, 135-142.

Knapp, T. J. (1976). A functional analysis of gambling behavior. En W. R. Edington (Ed.), Gambling and society (pp. 276-294). Springfield, IL: Charles C. Thomas.

Kowalski, R. M., Giumetti, G. W., Schroeder, A. N. y Lattanner, M. R. (2014). Bullying in the digital age: A critical review and meta-analysis of cyberbullying research among youth. Psychological Bulletin, 140 (4), 1073-1137.

Lapenta, F. (2011). Geomedia: On Location-Based Media, the Changing Status of Collective Image Production and the Emerging of Social Navigation Systems. Visual Studies, 26 (1), 14-24.

L'Ecuyer, C. (2015). Educar en la realidad. Barcelona: Plataforma Editorial.

L'Ecuyer, C. (26 de octubre de 2018). ¿Son nuestros alumnos nativos digitales? [Mensaje en un blog]. Recuperado de http://www.eduforics.com/ es/son-nuestros-alumnos-nativos-digitales/ (Consultado el 11/02/2019).

Lee, S. J. y Chae, Y. G. (2007). Children's Internet use in a family context: Influence on family relationships and parental mediation. Cyberpsychology \& Behavior, 10 (5), 640-644.

Lin, X., Zhou, H., Dong, G. y Du, X. (2015). Impaired risk evaluation in people with Internet gaming disorder: fMRI evidence from a probability discounting task. Progress in Neuro-Psychopharmacology and Biological Psychiatry, 56, 142-148.
Livingstone, S., Haddon, L., Görzig, A. y Ólafsson, K. (2011). Risks and safety on the Internet: The perspective of European children. Full findings and policy implications from the EU Kids Online survey of 9-16 year olds and their parents in 25 countries. London: EU Kids Online. Recuperado de http://eprints.lse.ac.uk/33731/ (Consultado el 11/02/2019).

Livingstone, S. y Helsper, E. J. (2008). Parental mediation of children's Internet use. Journal of Broadcasting \& Electronic Media, 52 (4), 581-599.

Livingstone, S. y Smith, P. K. (2014). Research Review: Harms experienced by child users of online and mobile technologies: The nature, prevalence and management of sexual and aggressive risks in the digital age. Journal of Child Psychology \& Psychiatry, 55, 635-654.

Loh, K. K. y Kanai, R. (2014). How has the Internet Reshaped human cognition? Neuroscientist, 22 (5), 506-520.

Marcum, C., Higgins, G. y Ricketts, M. (2010). Potential factors of online victimization of youth: An examination of adolescent online behaviors utilizing Routine Activities Theory. Deviant Behavior, 31 (5), 1-31.

Martellozzo, E. (2013). Online Child Sexual Abuse: Grooming, Policing and Child Protection in a Multi-Media World. London: Routledge.

Mesch, G. S. (2009). Parental mediation, online activities, and cyberbullying. Cyberpsychology and Behavior, 12 (4), 387-393.

Miller, D. (2018). Digital anthropology. The Cambridge Encyclopedia of Anthropology. Recuperado de http://www.anthroencyclopedia.com/ entry/digital-anthropology (Consultado el $11 / 02 / 2019)$.

Miró, F. (2012). El cibercrimen. Fenomenología y criminología de la delincuencia en el ciberespacio. Madrid: Marcial Pons.

Miró, F. y García, N. (2014). Ciberapp: estudio sobre el alcance de la cibercriminalidad contra menores en la provincia de Alicante. Alicante: Diputación de Alicante.

Mitchell, K., Finkelhor, D. y Wolak, J. (2001). Risk factors for and Impact of Online Sexual Solicitation of Youth. Journal of the American Medical Association, 285 (23), 3011-3014. 
Mitchell, K., Finkelhor, D. y Wolak, J. (2007). Youth Internet users at risk for the most serious online sexual solicitations. American Journal of Preventive Medicine, 32, 532-537.

Montiel, I. (2016). Cibercriminalidad social juvenil: La cifra negra. Revista d'Internet, Dret i Politic, 22, 119-131.

Montiel, I. (2018). Ciberacoso sexual en adolescentes: creencias erróneas. Revista Iberoamenricana de Psicología, 11 (2).

Montiel, I. y Agustina, J. R. (2018). Victimización sexual de menores a través de las TIC. En D. Dupuy (Dir.) y M. Kiefer (Coord.), Cibercrimen II (pp. 405-442). Buenos Aires: Editorial BdeF.

Montiel, I. y Carbonell, E. (2016). Vulnerabilidad y riesgo en los adolescentes: perfil del jugador de azar online. En E. Echeburúa (Coord.), Abuso de Internet. ¿Antesala para la adicción al juego de azar online? (pp. 169-190). Madrid: Ediciones Pirámide.

Montiel, I., Carbonell, E. y Pereda, N. (2016). Multiple Online Victimization of Spanish Adolescents: results from a community sample. Child Abuse and Neglect, 52, 123-134.

Montiel, I., Carbonell, E. y Salom, M. (2014). Victimización infantil sexual online: online grooming, ciber-abuso y ciber-acoso sexual. En M. Lameiras Fernández y E. Orts Berenguer (Coords.), Delitos sexuales contra menores. Abordaje psicológico, jurídico y policial (pp. 203-224). Valencia: Tirant Lo Blanch.

Moore, R., Guntupalli, N. T. y Lee, T. (2010). Parental regulation and online activities: Examining factors that influence a youth's potential to become a victim of online harassment. International Journal of Cyber Criminology, 4 (1/2), 685-698.

Navarro, R., Serna, C., Martínez, V. y Ruiz-Oliva, R. (2012). The role of Internet use and parental mediation on cyberbullying victimization among Spanish children from rural public schools. European Journal of Psychology of Education, 28 (3), 725-745.

Oliva, A., Antolín-Suárez, L., Ramos, P., Jiménez, L., Jiménez-Iglesias, A., Moreno, M. C. e Hidalgo, M. V. (2014). Adicciones con y sin sustancia: paralelismo. En M. T. Laespada y A. Estévez (Eds.), iExisten las adicciones sin sustancia? (pp. 87-99). Bilbao: Universidad de Deusto.
Ortega-Barón, J., Buelga, S. y Cava, M. J. (2016). Influencia del clima escolar y familiar en adolescentes, víctimas de ciberacoso. Comunicar, 24 (46), 57-65.

Ortega y Gasset, J. (1914). Meditaciones del Quijote. Madrid: Imprenta Clásica Española.

Osgood, D. W. y Anderson, A. L. (2004). Unstructured socializing and rates of delinquency. Criminology, 42 (3), 519-550.

Pereda, N., Guilera, G. y Abad, J. (2014). Victimization and polyvictimization of Spanish children and youth: Results from a community sample. Child Abuse \& Neglect, 38, 640-649.

Pink, S. y Hjorth, L. (2012). Emplaced Cartographies: Reconceptualising Camera Phone Practices in an Age of Locative Media. Media International Australia, 145 (1), 145-55.

Rettberg, J. W. (2014). Seeing Ourselves through Technology: How We Use Selfies, Blogs and Wearable Devices to See and Shape Ourselves. Basingstoke: Palgrave Macmillan.

Rowlands, I., Nicholas, D., Williams, P., Huntington, P., Fieldhouse, M., Gunter, B. y Tenopir, C. (2008). The Google generation: the information behaviour of the researcher of the future. Aslib proceedings, 60 (4), 290-310.

Sanjek, R. y Tratner, S. W. (2015). eFieldnotes: The makings of anthropology in the digital world. Philadelphia: University of Pennsylvania Press.

Silva Sánchez, J. Mํa (2018). La paradoja de la emancipación. Discurso pronunciado en la Ceremonia de inauguración del año académico 2018 de la Facultad de Derecho de la Pontificia Universidad Católica de Chile (130 aniversario). Santiago de Chile.

Sherman, L. E., Greenfield, P. M., Hernandez, L. M. y Dapretto, M. (2018). Peer influence via instagram: Effects on brain and behavior in adolescence and young adulthood. Child development, 89 (1), 37-47.

Sherman, L. E., Payton, A. A., Hernandez, L. M., Greenfield, P. M. y Dapretto, M. (2016). The power of the like in adolescence: Effects of peer influence on neural and behavioral responses to social media. Psychological Science, 27 (7), 1027-1035. 
Slonje, R., Smith, P. K. y Frisén, A. (2013). The nature of cyberbullying, and strategies of prevention. Computers in Human Behavior, 29, 26-32.

Smith, P. K. (2014). Understanding School Bullying: It's Nature and Prevention Strategies. Thousand Oaks, CA: Sage Publications.

Smith, P. K. y Livingstone, S. (2017). Child Users of Online and Mobile Technologies-Risks, Harms and Intervention. En D. Skuse, H. Bruce y L. Dowdney (Eds.), Child Psychology and Psychiatry: Frameworks for Clinical Training and Practice (pp. 141-148). Hoboken, NJ: John Wiley \& Sons, Inc.

Starcevic, V., Billieux, J. y Schimmenti, A. (2018). Selfitis, selfie addiction, twitteritis: irresistible appeal of medical terminology for problematic behaviours in the digital age. The Australian and New Zealand Journal of Psychiatry, 52 (5), 408-409.

Steeves, V. y Webster, C. (2008). Closing the barn door: the effect of parental supervision on Canadian children's online privacy. Bulletin of Science, Technology \& Society, 28 (1), 4-19.

Suler, J. (2004). The online disinhibition effect. Cyberpsychology \& behavior, 7 (3), 321-326.

Tacchi, J., Kitner, K. R. y Crawford, K. (2012). Meaningful Mobility. Feminist Media Studies, 12 (4), 528-537.

Tsitsika, A., Schoenmakers, T. M., Tzavela, E. C., Olafsson, K., Wójcik, S., Macarie, G. F., ... Richardson, C. (2014). Internet Addictive Behavior in Adolescence: A Cross-Sectional Study in Seven European Countries. CyberPsychology, Behavior \& Social Networking, 17 (8), 528-535.

Valcke, M., De Wever, B., Van Keer, H. y Schellens, T. (2011). Long-term study of safe Internet use of young children. Computers and Education, 57, 1292-1305.

Villacampa, C. (2017). Teen sexting: Prevalence, characteristics and legal treatment. International Journal of Law, Crime and Justice, 49, 10-21.

Walrave, M. y Heirman, W. (2011). Cyberbullying: Predicting victimisation and perpetration. Children \& Society, 25 (1), 59-72.

Wang, J., Iannotti, R. J. y Nansel, T. R. (2010). School bullying among adolescents in the United States: Physical, verbal, relational, and cyber. Journal of Adolescent Health, 45 (4), 368-375.
Webster, S., Davidson, J., Bifulco, A., Gottschalk, P., Caretti, V., Pham, T., ... Craparo G. (2012). Final Report-Executive Summary. European Online Grooming Project. Recuperado de http://www.crds.be/userfiles/files/European\%20 Online\%20Grooming\%20Project_Final\%20 Version_140312.pdf (Consultado el 11/02/2019). Wells, M. y Mitchell, K. J. (2008). How do high-risk youth use the Internet? Characteristics and implications for prevention. Child Maltreatment, 13 (3), 227-234.

Whittle, H., Hamilton-Giachritsis, C., Beech, A. y Collings, G. (2013). A review of young people's vulnerabilities to online grooming. Agression and Violent Behavior, 18, 135-146.

Wolak, J., Finkelhor, D. y Mitchell, K. (2004). Internet-initiated sex crimes against minors: Implications for prevention based on findings from a national study. Journal of Adolescent Health, 35 (5), 424-e11-424-e20.

Wolak, J., Mitchell, K. y Finkelhor, D. (2003). Escaping or connecting? Characteristics of youth who form close online relationships. Journal of Adolescence, 26 (1), 105-119.

Wolf, G. (1996). Steve Jobs: The Next Insanely Great Thing. Wired. Recuperado de https:// www.wired.com/1996/02/jobs-2/ (Consultado el 11/02/2019).

Yao, Y. W., Chen, P. R., Li, S., Wang, L. J., Zhang, J. T., Yip, S. W. y Fang, X. Y. (2015). Decision-making for risky gains and losses among college students with Internet gaming disorder. PloS one, 10 (1), e0116471. doi: https://doi. org/10.1371/journal.pone.0116471

Ybarra, M. y Mitchell, K. (2004). Youth engaging in online harassment: Associations with caregiver-child relationships, Internet use, and personal characteristics. Journal of Adolescence, 27, 319-336.

Ybarra, M., Mitchell, K. y Korchmaros, J. (2011). National Trends in Exposure to and Experiences of Violence on the Internet Among Children. Pediatrics, 128 (6), e1376-e1386. 


\section{Biografía de los autores}

Irene Montiel es Doctora en Psicología por la Universidad de Valencia, Licenciada en Psicología y en Criminología por la misma universidad y Máster en Psicología Jurídica por la Universidad Católica San Vicente Mártir. Actualmente es Profesora Ayudante Doctora en la Universidad Internacional de Catalunya donde coordina el Máster en Ciberdelincuencia. Entre sus líneas de investigación destaca la cibervictimización en menores.

(iD https://orcid.org/0000-0002-0537-2458
José R. Agustina es Profesor Agregado de Derecho Penal en la Universitat Internacional de Catalunya. Obtuvo el grado de Doctor en Derecho por la Universitat Pompeu Fabra y ha sido acreditado como Catedrático de Derecho Penal por AQU Catalunya. Actualmente dirige el Máster en Ciberdelincuencia y el Máster en Psicopatología Legal, Forense y Criminológica. Entre sus líneas de investigación principales, se ha especializado en la cibervictimización en menores.

(iD https://orcid.org/0000-0002-9254-6902 


\section{revista española de pedagogía \\ año 77, n 273, mayo-agosto 2019 \\ Spanish Journal of Pedagogy \\ year 77, n. 273, May-August 2019 \\ Sumario* \\ Table of Contents **}

\section{Respuestas educativas \\ y cívico-penales a los comportamientos antisociales}

\section{Educational and civic-penal responses to antisocial behavior}

Editor invitado: Fernando Gil Cantero Guest editor: Fernando Gil Cantero

\section{Fernando Gil Cantero}

Presentación: Respuestas educativas y cívico.penales a los comportamientos antisociales

Introduction: Educational and civic-penal responses to antisocial behavior

David Reyero, Fernando Gil Cantero

La educación que limita es la que libera

Education that limits is education that frees

Maria José Bernuz Beneitez, Esther Fernández Molina La pedagogía de la justicia de menores: sobre una justicia adaptada a los menores

The pedagogy of juvenile justice: a child-friendly justice

Marina Martins, Carolina Carvalho

¿En qué mienten los adolescentes?

What do teenagers lie about?
Inmaculada Méndez, Cecilia Ruiz Esteban, Juan Pedro Martínez, Fuensanta Cerezo

Ciberacoso según características sociodemográficas y académicas en estudiantes universitarios

Cyberbullying according to sociodemographic and academic characteristics among university students

261

Irene Montiel, José R. Agustina

Retos educativos ante los riesgos emergentes en el ciberespacio: claves para una adecuada prevención de la cibervictimización en menores

Educational challenges of emerging risks in cyberspace: foundations of an appropriate strategy for preventing online child victimization

Maialen Garmendia Larrañaga, Estefanía Jiménez Iglesias, Nekane Larrañaga Aizpuru Bullying y ciberbullying: victimización, acoso y daño.

Necesidad de intervenir en el entorno escolar Bullying and cyberbullying: victimisation, harassment, and harm. The need to intervene in the educational centre

Ana Rosser-Limiñana, Raquel Surià-Martínez Adaptación escolar y problemas comportamentales y emocionales en menores expuestos a violencia de género

School adaptation and behavioural and emotional problems in minors exposed to gender violence

\footnotetext{
* Todos los artículos están también publicados en inglés en la página web de la revista: https://revistadepedagogia.org.

** All the articles are also published in English on the web page of the journal: https://revistadepedagogia.org.
} 
Fanny T. Añaños-Bedriñana, Miguel Melendro, Rocío

Raya Miranda

Mujeres jóvenes con medidas de protección y judiciales y sus tránsitos hacia la prisión

Young women with protective and judicial measures and their transit towards prison

333

\section{Reseñas bibliográficas}

\section{Esteban Bara, F. Ética del profesorado (Juan} García Gutiérrez). García Amilburu, M., Bernal, A. y González Martín, M. R. Antropología de la educación. La especie educable (Yaiza Sánchez Pérez). Rose, D. y Martin, J. R. Leer para aprender. Lectura y escritura en las áreas del currículo (Francisco Lorenzo Bergillos). Buxarrais, M. R. y Vilafranca, I. (Coords.). Una mirada femenina de la educación moral (Eric Ortega González).

351

\section{Informaciones}

«ECER conference Education in an Era of Risk.the Role of Educational Research for the Futurey - de la European Educational Research Association (EERA)-; III Congreso «European Liberal Arts and Core Texts Educations; VIII Congreso del Jubilee Centre for Character and Virtues: «Virtues and the Flourishing Lifen; XLV Congreso Internacional de AME 2019: «Morality and Ethics for the Digital World»; III Premio Ricardo Marín Ibáñez; Una visita a la hemeroteca (profesores youtubers) (Daniel Pattier); Una visita a la red (David Reyero).

\section{Instrucciones para los autores}

Instructions for authors

375

ISSN: 0034-9461 (Impreso), 2174-0909 (Online)

https://revistadepedagogia.org/

Depósito legal: M. 6.020 - 1958

INDUSTRIA GRÁFICA ANZOS, S.L. Fuenlabrada - Madrid 


\section{Educational challenges of emerging risks in cybers- pace: foundations of an appropriate strategy for preventing online child victimisation*}

\section{Retos educativos ante los riesgos emergentes en el ciberespacio: claves para una adecuada prevención de la cibervictimización en menores}

Irene MONTIEL, PhD. Assistant Professor. Universitat Internacional de Catalunya (imontie@@uic.es). José R. AGUSTINA, PhD. Associate Professor (accredited as Full Professor). Universitat Internacional de Catalunya (jragustina@uic.es).

\section{Abstract:}

In just a few years, technological changes have transformed how people interact and communicate with each other, in particular among so-called digital adolescents. The impact of technology on routine activities and mainstream culture has led to an increase in young peoples' exposure to psychological and criminological risks. As a result of this new psychosocial trend, new educational challenges are appearing and it is becoming more necessary to react to these challenges on the basis of an adequate diagnosis of psychology and pedagogy relating to adolescents.

\footnotetext{
* This work was carried out under the framework of the research project "Criminology, empirical evidence and crime policy. Incorporating scientific data for decision making relating to the criminalisation of behaviour", reference: DER2017-86204-R, funded by Spain's State Research Agency (AEI)/Spanish Ministry of Science, Innovation, and Universities and the European Union through the European Regional Development Fund -ERDF — "Una manera de hacer Europa" (A way of making Europe).

Revision accepted: 2019-02-12.

This is the English version of an article originally printed in Spanish in issue 273 of the revista española de pedagogía. For this reason, the abbreviation EV has been added to the page numbers. Please, cite this article as follows: Montiel, I., \& Agustina, J. R. (2019). Retos educativos ante los riesgos emergentes en el ciberespacio: claves para una adecuada prevención de la cibervictimización en menores $\mid$ Educational challenges of emerging risks in cyberspace: foundations of an appropriate strategy for preventing online child victimisation. Revista Española de Pedagogía, 77 (273), 277-294. doi: https://doi.org/10.22550/REP77-2-2019-03
} 
Criminological theories and research have tried to identify risk and protection factors to understand victimisation processes in more depth and improve prevention strategies. In this context, it is necessary to develop educational programs that consider personal, familial, and situational vulnerabilities and weaknesses in order to foster resilient individuals who can successfully confront the risks inherent to cyberspace. By analysing specific forms of cybervictimisation, guidelines based on criminological research and the most frequent victimisation processes will be identified in order to improve design of educational programs focused on parents, educators and potential victims.

Keywords: cybervictimisation of minors, cyberpsychology, education in cyberspace, pedagogy of prosocial values, communicating to prevent crime, criminological theories useful for education, cyberbullying, grooming, sexting.

\section{Resumen:}

El avance tecnológico ha transformado en pocos años la forma de interactuar y comunicarse entre las personas, especialmente entre los denominados adolescentes digitales. Su impacto en las actividades cotidianas y en la cultura dominante ha propiciado, asimismo, un incremento en la exposición a riesgos psicológicos y criminológicos por parte de los menores. Ante esta nueva realidad psicosocial, se plantean nuevos retos educacionales que deben partir de un adecuado diagnóstico de la psicología y pedagogía en relación con los adolescentes.

Las teorías e investigaciones criminológicas vienen tratando de identificar los factores de riesgo y de protección con la finalidad de comprender con mayor profundidad los procesos de victimización y mejorar las estrategias de prevención. En este contexto, conviene avanzar en programas educativos que tengan en cuenta las carencias y vulnerabilidades personales, familiares y situacionales de los menores, a fin de fomentar el desarrollo de personas resilientes que sepan afrontar con éxito los riesgos que se fraguan y surgen en el ciberespacio. Mediante el análisis de las concretas formas de cibervictimización se pretende señalar algunas pautas basadas en la investigación criminológica y en los procesos de victimización más frecuentes en los entornos en que se desenvuelven los menores, de modo que puedan servir en el diseño de los programas educativos dirigidos a padres, educadores y potenciales víctimas.

Descriptores: cibervictimización en menores, ciberpsicología, educación en el ciberespacio, pedagogía en valores prosociales, comunicación para la prevención del delito, teorías criminológicas aplicables en educación, cyberbullying, grooming, sexting. 


\section{The anthropology of the smart- phone and criminological theories applied to adolescent cyber-victi- misation}

With the proliferation and spread of the use of ITCs in contemporary society, a new relational paradigm has established itself in personal and social interactions. The line separating the real-me from the digital-me is ever thinner and more patchy. Indeed, in people's everyday activities, and especially with so-called digital natives, communication is increasingly based on virtual media. Even the offline world and online reality intermingle, for example, through augmented reality. All of these changes in the physical world and its movement into cyberspace have caused major psychological, cultural, and, as will be shown below, victimological transformations.

As has been exposed in other publications, cyberspace and psychopathology are two dimensions that feed back into each other and, indeed, "a certain psychopathological symbiosis" sometimes occurs between aggressors and victims (Agustina, 2014; Gassó, Fernández-Cruz, Montiel, Martin-Fumadó, \& Agustina, 2018). "What elements of cyberspace lead to this weakening of the psychological barriers that block hidden feelings and needs?" asks Suler (2004, p. 322). Alongside other positive consequences, human beings in the digital era undoubtedly show greater vulnerability. Beyond transhumanism, it is worth focussing on the anthropological changes which technological changes produce in all dimensions of the sphere of human activity: perception, knowledge, learning, communication, interaction, and, ultimately, victimisation.
Miller (2018), when referring to the new field of study dubbed digital anthropology, states that greater emphasis in ethnographic analyses should be placed on those forms of digital culture that have become an omnipresent reality, such as social media networks and smartphones. Developing Ortega y Gasset's (1914) original idea, it is now possible to say, with regards to personal identity, that the I am I and my circumstances is strongly shaped by dependence on smartphones. And while the Internet was initially celebrated as a medium with unlimited freedom, this expectation has evolved, becoming about total monitoring and vigilance as the residents of the digital panopticon communicate intensively and bare themselves by their own free will (Han, 2014).

This era of full transparency has a major impact on the anthropological, psychological, and criminological study of the human being. As Favero and Theunissen (2018) note, digital technologies are entering anthropologists' lives as a digital logbook, in their fieldwork and in all of their activities, shaping how they record, process, analyze, and share their findings (Sanjek \& Tratner, 2015). Smartphones have, indeed, contributed to this Copernican turn as they have become an integral part of our day-to-day activities (Collins et al., 2017; Lapenta, 2011; Pink \& Hjorth, 2012; Tacchi, Kitner, \& Crawford, 2012): with these technologies even being implanted or embedded in the body itself, we are witnessing a progressive crossing between material bodies and mobile digital technologies (Favero, 2016; Ibrahim, 2015; Rettberg, 2014). 
The reduction in the age at which people access ITCs over recent years has involved very major changes in minors' dayto-day activities in cyberspace (García, 2017). They have virtual interactions outside the home much earlier and at all times, accessing these interactions with smartphones, which have become not just a window on the world, but also a real expanded space for their victimisation (Montiel \& Agustina, 2018).

Cyberspace is undoubtedly a new space for criminal opportunities (Miró, 2012) where children and young people continue to be protagonists, especially in the new forms of social cybercriminality (Miró, 2012), in other words, criminal phenomena which encompass different forms of online interpersonal victimisation, such as cyberbullying, online grooming, and unwanted sexting, which will be discussed below.

Criminological theories provide three decisive focusses in this digital era: the self-control approach (Gottfredson \& Hirschi, 1990), the routine activities approach (Cohen \& Felson, 1979), and the learning theories (Akers, 1990) among others. In fact, firstly, levels of self-control in the digital era are an individual factor of the first order, which make it possible to defer immediate rewards in a technological context which encourages impulsiveness. The tendency to develop addictive behaviour (for example, relating to compulsive consumption of all sorts of goods and services, including pornography, online gambling, and others) undoubtedly weakens the individual's will and resilience. Secondly, the everyday environment matters: environmental factors in the form of digital architectures and the design of online spaces that encourage anonymity and a lack of vigilance are factors that create opportunities for misuse, which aggressors use motivated by the limited perceived risk and effort in delinquent behaviour. Human beings' vulnerability largely depends on a lack of controls (capable guardians in the terms of the routine activities approach) and the ever greater amount of time young people spend connected, interacting with people they know and with strangers, unaware of the dangers that threaten them or aware of them but with a limited ability to weigh up their possible long-term consequences. Finally, learning theories can also explain how the influence of patterns of behaviour and inadequate models increases, and so negative values and messages about neutralisation of deviant behaviour emerge with great force, transforming the perceptions and values of the collective imagination and adolescent motivation.

\section{Cultural criminology and digi- tal adolescents: towards a snap- shot of youth culture and strength- ening digital resilience}

Katz already noted in his famous Seductions of Crime (1988) that the central issue when explaining how the decision to commit a crime arises lies in motivation and, specifically, in understanding how a "distinctive sensual dynamics" ( $p$. 4) emerges in the individual who commits it. The true nature of the human being is emotional: attention is sentiment and the conscience is sensual. The challenge when explaining this dynamic which encourages 
crime is to determine the steps in the dialectic process through which people empower the world to seduce them to commit a crime. And part of this challenge is discovered when recognising the different sequences in which this spirit of determinism is forged, sequences which are sufficiently subtle for their advances to go unnoticed (Katz, 1988).

If the key to explaining crime is emotional and aesthetic, the messages that should be sent to the homo sentimentalis to dissuade it from the pull of offending should be based on a visual language built by aesthetically underpinned prosocial values. In this sense, from cultural criminology an approach has emerged in recent times where the dimension of the image plays an increasingly central role. In effect, as Herrera notes (2014, p. 6), a new visual criminology is now appearing, with a marked iconic turn: if criminology studies crime, visual criminology would encompass the study of the modes in which the visual interacts with crime, with the two dimensions mutually shaping one another. Its essential objective is to inquire into the "modern visual aesthetic of criminality", insofar as it is intimately connected to a specific cultural ethic.

The new adolescent culture is a culture of the image transformed to unforeseen extremes by the technological setting, taking shape in the psychology of feedback, which has resulted in new forms or derivations of psychological disorders like a new digital narcissism or body dysmorphic disorder (Aiken, 2016). We have moved from the Kodak culture to the online image and the Instagram culture (Gómez, 2012), and the appearance of different labels like selfitis and twitteritis (Balakrishnan \& Griffiths, 2018; Starcevic, Billieux, \& Schimmenti, 2018). Users receive pleasurable input of explicit and quantifiable social approval (Sherman, Payton, Hernandez, Greenfield, \& Dapretto, 2016; Sherman, Greenfield, Hernandez, \& Dapretto, 2018), with unpredictable frequency and size, such as likes, views, or Google's search indicators (Loh \& Kanai, 2014), which reinforce the behaviour patterns of reward seeking and compulsive behaviour (Knapp, 1976). On the other hand, a growing number of studies have associated addictive behaviour relating to the Internet with changes in reward processing (e.g., Lin, Zhou, Dong, \& Du, 2015; Yao et al., 2015), processing of emotions, executive attention, decision making, impulse control (Oliva et al., 2013), and self-control mechanisms (Brand, Young, \& Laier, 2014; Greenfield, 2011). We face a paradox of emancipation (Silva, 2018): everything points to greater levels of emotional freedom, yet technology effervescence have produced an alarming atrophying of self-control capacities.

Perhaps the time has come to admit that the digital native concept is overvalued, as various authors claim (e.g., Kirschner \& De Bruyckere, 2017; L'Ecuyer, 2015; Rowlans et al., Nicholas, Williams, Huntington, Fieldhouse, Gunter, \& Tenopir, 2008). In this sense, Rowlans et al. (2008) recognise that while young people show great familiarity and technical agility with technology, they also depend too much on search engines and they lack the 
critical and analytic skills to be able to understand the value and originality of information on the Internet. Along the same lines, Carr (2011) affirms that digital natives gravitate towards a superficial way of processing information, characterised by rapid displacement of attention and minimal reflection.

\section{3. "As you sow, so shall you} reap": vulnerability, risk factors and protection, and new forms of victimisation

The effects of three social revolutions of great importance (the sexual revolution, the digital revolution, and the adolescent revolution), combined with the loss of the sense of privacy (Agustina, 2010 a; Agustina \& Gómez-Durán, 2016), , have had a particularly strong impact on digital minors. And so, with socio-cultural conditions conducive to overexposure to different types of risks having been created, new forms of victimisation have quickly appeared.

With this being the case, the different forms of online victimisation have adopted their own characteristics, leading to new forms of social criminality, such as cyberbullying, sexting, and online grooming (Miró, 2012). According to recent studies, over $50 \%$ of Spain's adolescent population has suffered at least one of these forms of social cybercriminality (Miró \& García, 2014; Montiel, Carbonell, \& Pereda, 2016), which can negatively affect young people's cognitive, neurological, and socio-affective development processes, increasing their risk of developing psychopathological dis- orders and behavioural problems, and increasing their vulnerability to victimisation in adulthood, as shown by numerous studies into victimisation of minors and polyvictimisation (Finkelhor, 2008; Finkelhor, Ormrod, \& Turner, 2007; Finkelhor, Turner, Ormrod, \& Hamby, 2009; Pereda, Guilera, \& Abad, 2014).

Cyberbullying between minors consists of repeated aggressive behaviour over time, intentionally carried out using electronic devices, with the aim of attacking a victim who cannot easily defend him or herself (Kowalski, Giumetti, Schroeder, \& Lattanner, 2014). This is a common phenomenon among young people in Spain and recent research shows that the prevalence between the ages of 12 and 18 is around $30-50 \%$ (Calvete, Orue, \& Gámez-Guadix, 2016). The invisibility and anonymity of the people involved and spectators, and the disinhibitory effect of this (Suler, 2004), the distance between victim and aggressor (especially emotional), the speed of dissemination of the content and the (near) impossibility of destroying it, the size of the audience, and its omnipresent nature are some of the elements that make this phenomenon an improved and more damaging version of traditional bullying. Harassment can come simultaneously from different channels to which the victim, aggressor, and spectators are constantly connected on different devices, in diverse contexts and situations, and so there are no safe spaces, not even the victim's own home (Kowalski et al., 2014). All of this not only promotes impulsive and disinhibited behaviour by the (ever more aggressive) perpetrators, 
but also facilitates the rapid isolation of the (ever more defenceless) victim and contributes to indefinitely prolonging his or her suffering and making the victimisation chronic (Slonje, Smith, \& Frisén, 2013) owing to the great difficulty of escaping and deleting the digital footprint (Montiel, 2016).

Online grooming is behaviour, generally carried out by an adult (or another minor who is significantly older than the victim) through the use of ICTs with the aim of misleading, manipulating, or deceiving a child for future online or offline sexual contact (Gámez-Guadix, Almendros, Calvete, \& De Santisteban, 2018). Montiel et al. (2016), based on a sample of 3,897 young people from Spain aged between 12 and 17, observe a prevalence of $17.2 \%$, with a higher rate of victimisation among girls. This increases to $25.6 \%$ with girls aged between 16 and 17 .

Grooming in itself does not necessarily involve sexual activity, but it does comprise the courting or seduction strategy used by the aggressor to approach minors, catch their attention and interest, seduce them, establish an affective bond with them, and reduce their inhibitions to increase the chance of success when making a sexual approach, as happens in traditional child sexual abuse (Montiel et al., 2016). Nonetheless, even in this process of seduction it is possible to fall back on sexual elements to reduce young people's inhibitions (showing sexual images of other minors or of adults, sexual conversations, etc.). The technological dimension of abuse now facilitates the groomer's process of preparation (observing and selecting victims, empowering and reinforcing their sexual interest in minors, etc.), as well as finding potential victims (concomitance of victims and settings), establishing a link, progressive sexualisation of the relationship (escalation and making the victim feel jointly responsible), and dissemination or interchange of visual evidence of the abuse (economic or social status gains in paedophile networks) (Gassó, Fernández-Cruz, Montiel, Martín-Fumadó, \& Agustina, 2018).

The term sexting is a portmanteau of "sex" and "texting", and the different definitions used include sending, receiving, or resending or disseminating sexually explicit messages or images of the protagonists in which they appear naked, semi-naked, or in a sexually suggestive form, with this sharing being done over mobile phones, social media, or the Internet (Agustina \& Montiel, 2016). In Spain, 33.5\% of adolescents practice sexting. It is most frequent among older adolescents (Villacampa, 2017) and it reaches its highest level among young adults (Gámez-Guadix, Almendros, Borrajo, \& Calvete, 2015). Some of the main complexities of this phenomenon revolve around the legal implications of the problem and the variety of content, behaviour, motivations, and media it includes (Drouin, Vogel, Surbey, \& Stills, 2013). Also in the significant debate over where to draw the line separating behaviour connected to sexual exploration between peers which can be regarded as normative from other types that are abusive and inappropriate (Livingstone \& Smith, 2014), which occur in aggressive or coer- 
cive contexts or involve child pornography. Only in these cases can we speak of sexting as a form of interpersonal online violence or a social cybercrime which also could well be the prelude to other cybercrimes such as cyberbullying or online grooming (Agustina \& Montiel, 2016).

Although it is important to note that the experience of these online risks does not always carry with it experience of harm (Livingstone, Haddon, Görzig, \& Ólafsson, 2011), over half of the victims of cyberbullying (Tsitsika et al., 2014) and at least one in three victims of unwanted online sexual experiences feels negatively affected by these experiences (Ybarra, Mitchell, \& Korchmaros, 2011), especially girls, younger children, and people with psychological difficulties (Livingstone \& Smith, 2014; Whittle et al., 2013). According to the review by Smith \& Livingstone (2017), the predictors for the appearance of harm deriving from experiences of online victimisation primarily refer to three groups: personality factors (sensation seeking, low self-esteem, moral disengagement, and psychological difficulties), social factors (lack of parental support and social standards), and digital factors (online practices or habits, digital skills, vulnerability to the lures of certain websites and services), and the authors suggest that interventions focus on these risk groups which are more vulnerable to mental harm, strengthening their capacity for resilience and coping strategies.

We should not ignore the fact that most of the adolescents involved in any of these forms of interpersonal online violence are also usually involved in others, giving rise to situations of multiple online victimisation, in the case of victims (Montiel et al., 2016), or general dysfunctional patterns of online behaviour in the case of aggressors (Montiel \& Carbonell, 2016), and that it is precisely this accumulation of negative experiences and behaviours which contributes to worse psychological and emotional adjustment of the minor (Pereda, Guilera, $\& \mathrm{Abad}, 2014$ ).

Among the factors present in groups of adolescents at risk, alongside certain characteristics of the young people themselves and the virtual space, there is a significant role for having relationships with their parents which are poor, conflictive, lack cohesion, or are negligent. From criminology, the family perspective is that widely held idea that a strong family reduces the existence of crime, while a weak family contributes to it appearing to a greater degree. Sampson has argued in this respect that family life (more than poverty in itself) is the main driving force in generating or avoiding crime. The family perspective fits in well with other common criminological perspectives. A favourable family environment tends to counteract human weaknesses, keeps young people away from unfavourable situations, keeps them away from bad company, from being inactive or idle, from undesirable temptations, risks, and provocations, while at the same time encouraging self-control when it is needed. Furthermore, theories of control tell us that children often fear embarrassing their parents by getting into problems. 
Family life also reduces the risk of victimisation (Agustina, 2010 b). In this sense, numerous studies identify family variables such as conflict and a lack of cohesion as predictors of cybervictimisation. For example, with regards to online grooming, Whittle, Hamilton-Giachritsis, Beech, \& Collings (2013) note that young people who are marginalised from their families, in conflict with their parents, or who have family difficulties are vulnerable to online sexual approaches (Mitchell, Finkelhor, \& Wolak, 2001, 2007; Wells \& Mitchell, 2008; Wolak, Finkelhor, \& Mitchell, 2004). Based on studies carried out with online groomers, it has been noted that they recognise young people who are looking for attention, empathy, or feedback from adults and they take advantage of these affective deficiencies (Webster et al., 2012; Santisteban \& Gámez-Guadix, 2017).

With regards to cyberbullying, parental support is an important protective factor (Wang, Iannotti, \& Nansel, 2010), while a poor parent/child relationship is a predictor of online bullying (Ybarra \& Mitchell, 2004). For their part, according to Baumgartner, Sumter, Peter, \& Valkenburg (2012), adolescents who are involved in risky online sexual behaviour like sexting, are less satisfied with their lives, display higher levels of sensation seeking, come from families with less cohesion and lower levels of education, and use the Internet more to communicate, supporting the idea that adolescents who have problems in their day-to-day lives can fall back on the Internet to substitute the loss of offline gratification (Wolak, Mitchell, \& Finkelhor, 2003).

\section{The culture of control versus the culture of education: recover- ing the concept of virtue through the paradigm of self-control}

We live in a society obsessed with control and with no tolerance for any type of risk, fixed in a prevention paradigm which does not appropriately understand that, in reality, education of the person and progressive learning in the exercise of a responsible freedom are far more decisive. Neither the state nor the modification of the social and cultural structures that encourages people to lead lifestyles where risks of victimisation are not sufficiently examined can guarantee protection of young people from the dangers of the digital era. External control mechanisms do not permeate the interior of the individual and, although the environment is decisive when shaping spaces for criminal opportunity, it is worth investing more in educating resilient people without ignoring the situational focus or modification of the socio-cultural structures mentioned above.

In this context, understanding the necessary gradualness with which the pedagogy of freedom must be implemented appears to be decisive. Prevention means, above all, education. And education in freedom should involve offering appropriate motivations for young people to see the positive and negative aspects of ITCs and choosing to opt for responsible use of technological resources. In any case, to educate it is necessary to supervise appropriately (Osgood \& Anderson, 2004). There are many ways of supervising: it is not just a case of accompanying, although 
the mere presence of parents and educators can undoubtedly favour more reflexive and prosocial behavioural dynamics. Some studies have analysed the efficacy of different parental mediation strategies on young people's Internet use, concluding that there is no simple and direct relationship between it and their online experiences. Therefore, merely increasing mediation does not reduce exposure to online risks (Livingstone \& Helsper, 2008) and the parental control perceived by the young people only minimally reduces their online risk-taking behaviour (Valcke, De Wever, Van Keer, \& Schellens, 2011).

Some authors maintain that parental control does not influence victimisation through cyberbullying (Marcum, Higgins, \& Ricketts, 2010; Moore, Guntupalli, \& Lee, 2010). Others, however, note that some specific parental supervision strategies can be protective factors, such as supervising time spent on the Internet and sharing of personal information (Ortega-Barón, Buelga, \& Cava, 2016; Walrave \& Heirman, 2011), while others, such as as monitoring pages they visit online, using filtering software, or the physical location of the computer do not work (Lee \& Chae, 2007; Mesch, 2009; Navarro, Serna, Martínez, \& Ruiz-Oliva, 2012). The impact of parental control depends, among other things, on how the young people use the Internet (Eynon \& Malmberg, 2011) and on how much this type of technology is embedded in their lives (Steeves \& Webster, 2008). In the first case, for example, the effect of parental supervision is greater on active users (all types of use, especially social) than on normative users (communication, entertainment, and looking for information) and peripheral users (limited use of the Internet) than on any other type of user (Eynon \& Malmberg, 2011).

Along with the presence of capable guardians (Hollis, Felson, \& Welsh, 2013) in the surroundings of the day-to-day activities of the young people, it is necessary to construct a communicative language which is effective in the context of the prevention programmes and instruments. And this language which transmits prosocial values, in cyberspace as well, must come from the family as the individual's primary location of socialisation and from other authority figures which contribute to forming the culture of the group or the social culture. The models which are genuinely successful in adolescent culture have a fundamental weight in the configuration of the set of messages favourable or unfavourable to risk practices.

The current crisis of values that afflicts us (libertarian antisocial behaviour, moral detachment or insensitivity, high levels of interpersonal violence, a tendency towards atomising individualist egotism, lack of business ethics, among other symptoms) is a reaction to two problems which feed into each other: a dimming of the intelligence to know good and a weakening of the will to decide to practice it. In light of the first problem, a return to the concept of virtue and the challenge of making it attractive in educational, political, and social discourse should be proposed; in light 
of the second problem, formulas should be proposed for re-strengthening the family structure as the ideal environment for developing the capacity for resilience and self-control. Some dominant lines of thought have placed the emphasis on a concept of unlimited freedom (apart from some minimal limits which, in view of the current situation, seem insufficient). Accordingly, a pedagogical model has been favoured, based on a certain self-justifying ethical emotivism. In this context, there should be a return to a positive concept of what it means to educate, which proposes purposefully returning to an emphasis on the importance of self-control as a counterweight to simply allowing oneself to be carried along by a flood of emotions, impulses, and objectives which are gratifying in the short term. The "self-control" construct is more than the contemporary translation of the Aristotelian concept of virtue.

From this perspective, we live in an anomic society where rules only fulfil an external role, of threatening or psychological coercion in the case of deviation. The lack of basic objective references in moral action and in dominant political and social discourse underlines the magnitude of the crisis of values affecting us. A certain existential pessimism stands out among the signs of disquiet of the postmodern individual, the result of a relativism of values which, prima facie, was going to bring us freedom and tolerance. This sensation of anguish and anxiety is certainly a paradoxical situation in the period in the history of humankind with the greatest well-being and security. The model of hap- piness based on compulsive hedonism has only increased the existential vacuum and lack of meaning of a society which, more than ever, is feeling in the dark. Even so, starting from an objective snapshot without euphemisms, efforts should be made to build a promising setting which returns to the family, educational authorities, means of social communication, and political leaders this vital desire to make effective improvements to the conditions for development in order to create responsible citizens.

Starting from these premises, training programmes for parents and educators should be directed towards a new culture in the use of ICTs which advocates a firm commitment to self-control as a counterpoint to the disinhibitory effects described by Suler (2004), empathy and rules for education in cyberspace (netiquette), moderation in habits for using the Internet, recovering the sense of privacy, awareness of the negative effects of narcissistic self-referentiality, or people's awareness of the consequences and the indelible trace of their online behaviour. At a more specific level, prevention of cyberbullying, for example, can focus on general training in empathy, modifying beliefs that support aggression, and establishing more specific guidelines for behaviour on the Internet, including actions which young people can take such as reporting abuse and collecting evidence (Smith \& Livingstone, 2017). Prevention of sexting can focus on sexual-affective education and emotional self-regulation, respect for one's own privacy and that of other people, and training about digital architecture and risks for 
online privacy which explain how we lose control of anything we publish online. For its part, preventing online grooming situations can primarily be based on reinforcing parental support and family cohesion, promoting healthy affective relationships which are not coercive or asymmetrical, and developing critical thinking about the hyper-sexualisation of childhood, dismantling all of the false beliefs and fallacies that make it more likely young people will find themselves involved in abusive online relationships (Montiel, Carbonell, \& Salom, 2014).

It is important to note that in cyberspace, the victim-aggressor dichotomy is becoming increasingly flexible, and it is even possible to speak of a continuum of involvement in which the figure of the aggressive-victim or the victimised-aggressor often appears (Montiel, 2016; Walrave \& Heirman, 2011) as there is enough empirical evidence to support a significant overlap of roles, both online and offline (Kowalski et al., 2014; Smith, 2014). Similarly, we know that children who are vulnerable offline are also vulnerable online, in the same way that people who take risks in one field are more likely to take them in others ones as well (Livingstone \& Smith, 2014). Accordingly, from a developmental victimology focus, Pereda et al. (2014) note an increased association between polyvictimisation and victimisation (physical and psychological) by carers, sexual victimisation (especially by unknown adults), and online victimisation (non-sexual cyberbullying and unwanted sexual approaches).
All of this points to the need to adopt environmental and integrated approaches which, instead of focussing on preventing specific behaviour patterns, such as cyberbullying or online grooming or on specific roles (victim or aggressor) or on microsystem levels of analysis (school or family), adopt a broader vision of online victimisation of children and adolescents in which, for many children, digital violence can be a virtually chronic condition of life rather than an isolated experience, which points to the presence of a strong structural root and indicates the existence of a serious endemic problem in current society.

In general, it is necessary to adapt prevention messages to make them suitable for adolescents, increase the degree of warning about risks, and motivate them to reconsider coming into contact with people they do not know online to offset their psychological weaknesses or shortcomings, such as loneliness or depression (Wolak et al., 2004), as well as allowing them to develop their capacity for self-regulation and self-control so they do not succumb to disinhibition, loss of control and freedom online, at the same time as strengthening protective factors that minimise the impact of risks. But to do this, it is necessary to offer them alternative more attractive and exciting spaces for development, ones that are more authentic than screens.

To prevent childhood and youth cyber-victimisation it is not enough to educate in technological skills, since, as L'Ecuyer (2018) notes, true preparation 
for using technologies correctly lies in understanding context, which does not develop in a decontextualised setting like the online one, but which is acquired in the offline setting, which is the real world. Neither can it be expected that technological applications themselves will provide a solution to the problem, given that it transcends the digital architecture of cyberspace, and we can be sure that what puts minors at risk is not computers or technology per se, but rather people (Martellozzo, 2013) and we believe that, as Steve Jobs said, "what's wrong with education cannot be fixed with technology" (Wolf, 1996).

\section{References}

Agustina, J. R. (2010 a). ¿Menores infractores 0 víctimas de pornografía infantil? Respuestas legales e hipótesis criminológicas ante el Sexting. Revista Electrónica de Ciencia Penal y Criminología, 12 (11), 1-44.

Agustina, J. R. (2010 b). Conceptos clave, fenomenología, factores y estrategias en el marco de la violencia intrafamiliar. In J. R. Agustina (Ed.), Violencia intrafamiliar. Raíces, factores y formas de la violencia en el hogar (pp. 61-132). Madrid: BdeF-Edisofer.

Agustina, J. R. (2014). Cibercriminalidad y perspectiva victimológica: un enfoque general explicativo de la cibervictimización. Cuadernos de Política Criminal, 114 (3), 143-178.

Agustina, J. R., \& Gómez-Durán, E. L. (2016). Factores de riesgo asociados al sexting como umbral de diversas formas de victimización. Estudio de factores correlacionados con el sexting en una muestra universitaria. Revista de Internet, Derecho y Política, 22, 21-47.

Agustina, J. R., \& Montiel, I. (2017). Sexting en adolescentes: nuevos retos médico-legales. Revista Española de Medicina Legal, 43 (1), 43-44.
Aiken, M. (2017). The Cyber Effect: An Expert in Cyberpsychology explains how Technology is Shaping Our Children, Our Behavior, and Our Value and what We Can Do about it. New York: Spiegel \& Grau.

Akers, R. L. (1990). Rational choice, deterrence, and social learning theory in criminology: The path not taken. Journal of Criminal Law \& Criminology, 81, 653-676.

Balakrishnan, J., \& Griffiths, M. D. (2018). An Exploratory Study of "Selfitis" and the Development of the Selfitis Behavior Scale. International Journal of Mental Health and Addiction, 16 (3), 722-736.

Baumgartner, S. E., Sumter, S. R., Peter, J., \& Valkenburg, P. M. (2012). Identifying Teens at Risk: Developmental Pathways of Online and Offline Sexual Risk Behavior. Pediatrics, 130 (6), 1489-1496. doi: https://doi.org/10.1542/ peds.2012-0842

Brand, M., Young, K. S., \& Laier, C. (2014). Prefrontal control and Internet addiction: a theoretical model and review of neuropsychological and neuroimaging findings. Frontiers in human neuroscience, 8, 1-13. doi: https://doi. org/10.3389/fnhum.2014.00375

Calvete, E., Orue, I., \& Gámez-Guadix, M. (2016). Cyberbullying Victimization and Depression in Adolescents: The Mediating Role of Body Image and Cognitive Schemas in a One-year Prospective Study. European Journal on Criminal Policy and Research, 22 (2), 271-284.

Carr, N. (2011). The shallows: what the Internet is doing to our brains. New York: WW Norton.

Cohen, L. E., \& Felson, M. (1979). Social Change and Crime Rate Trends: A Routine Activity Approach. American Sociological Review, 44 (4), 588-608.

Collins, S. G., Durington. M., Favero, P., Harper, K., Kenner, A., \& O'Donnell, C. (2017). Ethnographic Apps/Apps as Ethnography. Anthropology Now, 9 (1), 102-18.

De Santisteban, P., \& Gámez-Guadix, M. (2017). Estrategias de persuasión en grooming online de menores: Un análisis cualitativo con agresores en prisión. Psychosocial Intervention, 26 (3), 139-146. 
Drouin, M., Vogel, K. N., Surbey, A., \& Stills, J. R. (2013). Let's talk about sexting, baby: Computer mediated behaviors among young adults. Computers in Human Behavior, 29, A25-A26.

Eynon, R., \& Malmberg, L. E. (2011). A typology of young people's Internet use: implications for education. Computers and Education, 56 (3), 585-595.

Favero, P. (2016). Analogization: Reflections on Life-Logging Cameras, Action Cams and Images' Changing Meaning in a Digital Landscape. In E. G. Cruz, E. Lehmuskallio, \& A. Lehmuskallio (Eds.), Digital Photography and Everyday Life: Empirical Studies on Material Visual Practices (pp. 209-27). New York: Routledge.

Favero, P. S., \& Theunissen, E. (2018). With the Smartphone as Field Assistant: Designing, Making, and Testing EthnoAlly, a Multimodal Tool for Conducting Serendipitous Ethnography in a Multisensory World. American Anthropologist, 120 (1), 163-167.

Finkelhor, D. (2008). Childhood Victimization. Violence, Crime, and Abuse in the Lives of Young People. Oxford, USA: Oxford University Press.

Finkelhor, D., Ormrod, R. K., \& Turner, H. (2007). Revictimization patterns in a national longitudinal sample of children and youth. Child Abuse \& Neglect, 31, 479-502.

Finkelhor, D., Turner, H., Ormrod, R., \& Hamby, S. (2009). Violence, Abuse, and Crime Exposure in a National Sample of Children and Youth. Pediatrics, 124 (5), 1411-1423.

Gámez-Guadix, M., Almendros, C., Borrajo, E., $\&$ Calvete, E. (2015). Prevalence and association of sexting and online sexual victimization among Spanish adults. Sexuality Research and Social Policy, 12 (2), 145-154.

Gámez-Guadix, M., Almendros, C., Calvete, E., \& de Santisteban, P. (2018). Persuasion strategies and sexual solicitations and interactions in online sexual grooming of adolescents: Modeling direct and indirect pathways. Journal of Adolescence, 63, 11-18.

García Guilabert, N. (2017). El ciberacoso. Análisis de la victimización en menores en el ciberespacio desde la teoría de las actividades cotidianas. Madrid: Editorial B de F.
Gassó, A. M., Fernández-Cruz, V., Montiel, I., Martin-Fumadó, C., \& Agustina, J. R. (2018). Retos forenses ante la cibercriminalidad social en menores. Revista Española de Medicina Legal. doi: https://doi.org/10.1016/j.reml.2018.11.003

Gómez Cruz, E. (2012). De la cultura Kodak a la imagen en red: una etnografía sobre fotografía digital, 23. Barcelona: Editorial UOC.

Gottfredson, M. R., \& Hirschi, T. (1990). A general theory of crime. Palo Alto, CA: Stanford University Press.

Greenfield, D. (2011). The addictive properties of Internet usage. In K. S. Young, C. N. de Arbeu (Eds.), Internet addiction: a handbook and guide to evaluation and treatment (pp. 135-53). Hoboken, NJ: John Wiley.

Han, B. C. (2014). Psicopolítica. Barcelona: Herder. Herrera Moreno, M. (2014). Construcción cultural y prevención criminal publicista: Una revisión de casos conflictivos. Revista Electrónica de Ciencia Penal y Criminología, 16-10, 10:1-10:48.

Hollis, M. E., Felson, M., \& Welsh, B. C. (2013). The capable guardian in routine activities theory: A theoretical and conceptual reappraisal. Crime Prevention and Community Safety, 15 (1), 65-79.

Ibrahim, Y. (2015). Instagramming Life: Banal Imaging and the Poetics of the Everyday. Journal of Media Practice, 16 (1), 42-54.

Katz, J. (1988). Seductions of crime: Moral and sensual attractions in doing evil. New York: Basic Books.

Kirschner, P., \& de Bruyckere, P. (2017). The myths of the digital native and the multitasker. Teaching and Teacher Education, 67, 135-142.

Knapp, T. J. (1976). A functional analysis of gambling behavior. In W. R. Edington (Ed.), Gambling and society (pp. 276-294). Springfield, IL: Charles C. Thomas.

Kowalski, R. M., Giumetti, G. W., Schroeder, A. N., \& Lattanner, M. R. (2014). Bullying in the digital age: A critical review and meta-analysis of cyberbullying research among youth. Psychological Bulletin, 140 (4), 1073-1137.

Lapenta, F. (2011). Geomedia: On Location-Based Media, the Changing Status of Collective Image Production and the Emerging of Social Navigation Systems. Visual Studies, 26 (1), 14-24. 
L'Ecuyer, C. (2015). Educar en la realidad. Barcelona: Plataforma Editorial.

L'Ecuyer, C. (2018). ¿Son nuestros alumnos nativos digitales? EDUForics. Retrieved from http://www.eduforics.com/es/son-nuestrosalumnos-nativos-digitales/ (Consulted on $11 / 02 / 2019)$.

Lee, S. J., \& Chae, Y. G. (2007). Children's Internet use in a family context: Influence on family relationships and parental mediation. Cyberpsychology \& Behavior, 10 (5), 640-644.

Lin, X., Zhou, H., Dong, G., \& Du, X. (2015). Impaired risk evaluation in people with Internet gaming disorder: fMRI evidence from a probability discounting task. Progress in Neuro-Psychopharmacology and Biological Psychiatry, 56, 142-148.

Livingstone, S., Haddon, L., Görzig, A., \& Ólafsson, K. (2011). Risks and safety on the internet: The perspective of European children. Full findings and policy implications from the EU Kids Online survey of 9-16 year old and their parents in 25 countries. London: EU Kids Online. Retrieved from http://eprints.lse.ac.uk/33731/

Livingstone, S., \& Helsper, E.J. (2008). Parental mediation of children's Internet use. Journal of Broadcasting \& Electronic Media, 52 (4), 581-599.

Livingstone, S., \& Smith, P. K. (2014) Research Review: Harms experienced by child users of online and mobile technologies: The nature, prevalence and management of sexual and aggressive risks in the digital age. Journal of Child Psychology \& Psychiatry, 55, 635-654.

Loh, K. K., \& Kanai, R. (2014). How has the Internet Reshaped human cognition? Neuroscientist, 22 (5), 506-520.

Marcum, C., Higgins, G., \& Ricketts, M. (2010). Potential factors of online victimization of youth: An examination of adolescent online behaviors utilizing Routine Activities Theory. Deviant Behavior, 31 (5), 1-31.

Martellozzo, E. (2013). Online Child Sexual Abuse: Grooming, Policing and Child Protection in a Multi-Media World. London: Routledge.

Mesch, G. S. (2009). Parental mediation, online activities, and cyberbullying. Cyberpsychology and Behavior, 12 (4), 387-393.
Miller, D. (2018). Digital anthropology. Cambridge Encyclopedia of Anthropology. Retrieved from http://www.anthroencyclopedia.com/ entry/digital-anthropology (Consulted on 11/02/2019).

Miró, F. (2012). El cibercrimen. Fenomenología y criminología de la delincuencia en el ciberespacio. Madrid: Marcial Pons.

Miró, F., \& García, N. (2014). Ciberapp: estudio sobre el alcance de la cibercriminalidad contra menores en la provincia de Alicante. Alicante: Diputación de Alicante.

Mitchell, K., Finkelhor, D., \& Wolak, J. (2001). Risk factors for and Impact of Online Sexual Solicitation of Youth. Journal of the American Medical Association, 285 (23), 3011-3014.

Mitchell, K., Finkelhor, D., \& Wolak, J. (2007). Youth internet users at risk for the most serious online sexual solicitations. American Journal of Preventive Medicine, 32, 532-537.

Montiel, I. (2016). Cibercriminalidad social juvenil: La cifra negra. Revista d'Internet, Dret i Polític, $22,119-131$.

Montiel, I. (2018). Ciberacoso sexual en adolescentes: creencias erróneas. Revista Iberoamenricana de Psicología, 11 (2).

Montiel, I., \& Agustina, J. R. (2018). Victimización sexual de menores a través de las TIC. In D. Dupuy (Dir.) \& M. Kiefer (Coord.), Cibercrimen II (pp. 405-442). Buenos Aires: Editorial BdeF.

Montiel, I., \& Carbonell, E. (2016). Vulnerabilidad y riesgo en los adolescentes: perfil del jugador de azar online. In E. Echeburúa (Coord.), Abuso de Internet. ¿Antesala para la adicción al juego de azar online? (pp. 169-190). Madrid: Ediciones Pirámide.

Montiel, I., Carbonell, E., \& Pereda, N. (2016). Multiple Online Victimization of Spanish Adolescents: results from a community sample. Child Abuse and Neglect, 52, 123-134.

Montiel, I., Carbonell, E., \& Salom, M. (2014). Victimización infantil sexual online: online grooming, ciber-abuso y ciber-acoso sexual. In M. Lameiras Fernández, \& E. Orts Berenguer (Coords.), Delitos sexuales contra menores. Abordaje psicológico, jurídico y policial (pp. 203-224). Valencia: Tirant Lo Blanch. 
Moore, R., Guntupalli, N. T., \& Lee, T. (2010). Parental regulation and online activities: Examining factors that influence a youth's potential to become a victim of online harassment. International Journal of Cyber Criminology, 4 (1/2), 685-698.

Navarro, R., Serna, C., Martínez, V., \& Ruiz-Oliva, R. (2012). The role of Internet use and parental mediation on cyberbullying victimization among Spanish children from rural public schools. European Journal of Psychology of Education, 28 (3), 725-745.

Oliva, A., Antolín-Suárez, L., Ramos, P., Jiménez, L., Jiménez-Iglesias, A., Moreno, M. C., \& Hidalgo, M. V. (2014). Adicciones con y sin sustancia: paralelismo. In M. T. Laespada, \& A. Estévez (Eds.), ¿Existen las adicciones sin sustancia? (pp. 87-99). Bilbao: Universidad de Deusto.

Ortega-Barón, J., Buelga, S., \& Cava, M. J. (2016). Influencia del clima escolar y familiar en adolescentes, víctimas de ciberacoso. Comunicar, 24 (46), 57-65.

Ortega y Gasset, J. (1914). Meditaciones del Quijote. Madrid: Imprenta Clásica Española.

Osgood, D. W., \& Anderson, A. L. (2004). Unstructured socializing and rates of delinquency. Criminology, 42 (3), 519-550.

Pereda, N., Guilera, G., \& Abad, J. (2014). Victimization and polyvictimization of Spanish children and youth: Results from a community sample. Child Abuse \& Neglect, 38, 640-649.

Pink, S., \& Hjorth, L. (2012). Emplaced Cartographies: Reconceptualising Camera Phone Practices in an Age of Locative Media. Media International Australia, 145 (1), 145-55.

Rettberg, J. W. (2014). Seeing Ourselves through Technology: How We Use Selfies, Blogs and Wearable Devices to See and Shape Ourselves. Basingstoke: Palgrave Macmillan.

Rowlands, I., Nicholas, D., Williams, P., Huntington, P., Fieldhouse, M., Gunter, B., \& Tenopir, C. (2008). The Google generation: the information behaviour of the researcher of the future. Aslib proceedings, 60 (4), 290-310.

Sanjek, R., \& Tratner, S. W. (2016). eFieldnotes: The makings of anthropology in the digital world. Philadelphia: University of Pennsylvania Press.
Silva Sánchez, J. Mํㅡ. (2018). La paradoja de la emancipación. Discourse given in the 2018 academic year at the inauguration ceremony of the Faculty of Law at the Pontifical Catholic University of Chile ( $130^{\text {th }}$ anniversary). Santiago de Chile.

Sherman, L. E., Greenfield, P. M., Hernandez, L. M., \& Dapretto, M. (2018). Peer influence via instagram: Effects on brain and behavior in adolescence and young adulthood. Child development, 89 (1), 37-47.

Sherman, L. E., Payton, A. A., Hernandez, L. M., Greenfield, P. M., \& Dapretto, M. (2016). The power of the like in adolescence: Effects of peer influence on neural and behavioral responses to social media. Psychological Science, 27 (7), 1027-1035.

Slonje, R., Smith, P. K., \& Frisén, A. (2013). The nature of cyberbullying, and strategies of prevention. Computers in Human Behavior, 29, 26-32.

Smith P. K. (2014). Understanding School Bullying: It's Nature and Prevention Strategies. Thousand Oaks, CA: Sage Publications.

Smith, P. K., \& Livingstone, S. (2017). Child Users of Online and Mobile Technologies-Risks, Har$\mathrm{ms}$ and Intervention. In D. Skuse, H. Bruce, \& L. Dowdney (Eds.), Child Psychology and Psychiatry: Frameworks for Clinical Training and Practice (pp.141-148). Hoboken, NJ: John Wiley \& Sons, Inc.

Starcevic, V., Billieux, J., \& Schimmenti, A. (2018). Selfitis, selfie addiction, twitteritis: irresistible appeal of medical terminology for problematic behaviours in the digital age. The Australian and New Zealand Journal of Psychiatry, 52 (5), 408-409.

Steeves, V., \& Webster, C. (2008). Closing the barn door: the effect of parental supervision on Canadian children's online privacy. Bulletin of Science, Technology \& Society, 28 (1), 4-19.

Suler, J. (2004). The online disinhibition effect. $C y$ berpsychology \& behavior, 7 (3), 321-326.

Tacchi, J., Kitner, K. R., \& Crawford, K. (2012). Meaningful Mobility. Feminist Media Studies, 12 (4), 528-537. doi: https://doi.org/10.1080/14680 777.2012 .741869 
Tsitsika, A., Schoenmakers, T. M., Tzavela, E. C., Olafsson, K. Wójcik, S., Macarie, G. F., ... Richardson, C. (2014). Internet Addictive Behavior in Adolescence: A Cross-Sectional Study in Seven European Countries. CyberPsychology, Behavior \& Social Networking, 17 (8), 528-535.

Valcke, M., De Wever, B., Van Keer, H., \& Schellens, T. (2011). Long-term study of safe Internet use of young children. Computers and Education, 57, 1292-1305.

Villacampa, C. (2017). Teen sexting: Prevalence, characteristics and legal treatment. International Journal of Law, Crime and Justice, 49, 10-21.

Walrave, M., \& Heirman, W. (2011). Cyberbullying: Predicting victimisation and perpetration. Children \& Society, 25 (1), 59-72.

Wang, J., Iannotti, R. J., \& Nansel, T. R. (2010). School bullying among adolescents in the United States: Physical, verbal, relational, and cyber. Journal of Adolescent Health, 45 (4), 368375 .

Webster, S., Davidson, J., Bifulco, A., Gottschalk, P., Caretti, V., Pham, T., ... Craparo G. (2012). Final Report-Executive Summary. European Online Grooming Project. Retrieved from http://www.crds.be/userfiles/ files/European\%200nline\%20Grooming\%20 Project_Final\%20Version_140312.pdf (Consulted on $11 / 02 / 2019$ ).

Wells, M., \& Mitchell, K. J. (2008). How do high-risk youth use the Internet? Characteristics and implications for prevention. Child Maltreatment, 13 (3), 227-234.

Whittle, H., Hamilton-Giachritsis, C., Beech, A., \& Collings, G. (2013). A review of young people's vulnerabilities to online grooming. Aggression and Violent Behavior, 18, 135-146.

Wolak, J., Finkelhor, D., \& Mitchell, K. (2004). Internet-initiated sex crimes against minors: Implications for prevention based on findings from a national study. Journal of Adolescent Health, 35 (5), 424-e11-424-e20.

Wolak, J., Mitchell, K., \& Finkelhor, D. (2003). Escaping or connecting? Characteristics of youth who form close online relationships. Journal of Adolescence, 26 (1), 105-119.
Wolf, G. (1996). Steve Jobs: The Next Insanely Great Thing. Wired. Retrieved from https:// www.wired.com/1996/02/jobs-2/ (Consulted on 11/02/2019).

Yao, Y. W., Chen, P. R., Li, S., Wang, L. J., Zhang, J. T., Yip, S. W., \& Fang, X. Y. (2015). Decision-making for risky gains and losses among college students with Internet gaming disorder. PloS one, 10 (1), e0116471. doi: https://doi. org/10.1371/journal.pone.0116471

Ybarra, M., \& Mitchell, K. (2004). Youth engaging in online harassment: Associations with caregiver-child relationships, internet use, and personal characteristics. Journal of Adolescence, 27, 319-336.

Ybarra, M., Mitchell, K., \& Korchmaros, J. (2011). National Trends in Exposure to and Experiences of Violence on the Internet Among Children. Pediatrics, 128 (6), e1376-e1386.

\section{Authors' biographies}

Irene Montiel has a doctorate in Psychology from the Universidad de Valencia, an undergraduate degree in Psychology and Criminology from the same university, and a Master's in Legal Psychology from the Universidad Católica San Vicente Mártir. She is currently an Assistant Professor at the Universidad Internacional de Catalonia where she coordinates the Master's in Cybercrime. Cyber-victimisation in minors is one of her main research interests.

iD https://orcid.org/0000-0002-0537-2458

José R. Agustina is Associate Professor in Criminal Law at the Universitat Internacional of Catalonia (Barcelona, Spain). He gained a doctorate in Law at Universitat Pompeu Fabra and has been accredited as a Full Professor in Criminal Law by AQU Catalunya, the Agency for the Quality of the University system of Catalonia. He currently directs the Mas- 
ter's in Cybercrime and the Master's in Legal, Forensic, and Criminological Psychopathology. His research interests include specialising in cyber-victimisation of minors.

(D) https://orcid.org/0000-0002-9254-6902 
revista española de pedagogía año 77, n 273, mayo-agosto 2019

Spanish Journal of Pedagogy

year 77, n. 273, May-August 2019

\section{Table of Contents}

Sumario

\section{Educational and civic-penal responses to antisocial behavior}

Respuestas educativas y cívicopenales a los comportamientos antisociales

Guest editor: Fernando Gil Cantero

Editor invitado: Fernando Gil Cantero

\section{Fernando Gil Cantero}

Introduction: Educational and civic.penal responses to antisocial behavior

Presentación: Respuestas educativas y cívico-penales a los comportamientos antisociales

David Reyero, Fernando Gil Cantero

Education that limits is education that frees

La educación que limita es la que libera

Maria José Bernuz Beneitez, Esther Fernández Molina The pedagogy of juvenile justice: a child.friendly justice

La pedagogía de la justicia de menores: sobre una justicia adaptada a los menores
Marina Martins, Carolina Carvalho

What do teenagers lie about?

¿En qué mienten los adolescentes?

245
Inmaculada Méndez, Cecilia Ruiz Esteban, Juan Pedro Martinez, Fuensanta Cerezo

Cyberbullying according to sociodemographic and academic characteristics among university students Ciberacoso según características sociodemográficas y académicas en estudiantes universitarios

Irene Montiel, José R. Agustina

Educational challenges of emerging risks in cyberspace: foundations of an appropriate strategy for preventing online child victimization

Retos educativos ante los riesgos emergentes en el ciberespacio: claves para una adecuada prevención de la cibervictimización en menores

Maialen Garmendia Larrañaga, Estefanía Jiménez Iglesias, Nekane Larrañaga Aizpuru

Bullying and cyberbullying: victimisation, harassment, and harm. The need to intervene in the educational centre Bullying y ciberbullying: victimización, acoso y daño.

Necesidad de intervenir en el entorno escolar 


\section{Ana Rosser-Limiñana, Raquel Suriá-Martínez}

School adaptation and behavioural and emotional

problems in minors exposed to gender violence

Adaptación escolar y problemas comportamentales y

emocionales en menores expuestos a violencia de género

\section{Fanny T. Añaños-Bedriñana, Miguel Melendro}

\section{Estefanía, Rocio Raya Miranda}

Young women with protective and judicial measures

and their transit towards prison

Mujeres jóvenes con medidas de protección y judiciales y sus

tránsitos hacia la prisión

\section{Book reviews}

Esteban Bara, F. Ética del profesorado [Ethics of

teaching staff] (Juan García Gutiérrez). García

Amilburu, M., Bernal, A., \& González Martín, M.

313

R. Antropología de la educación. La especie educable [Anthropology of education. The educatable species] (Yaiza Sánchez Pérez). Rose, D., \& Martin, J. R. Leer para aprender. Lectura y escritura en las áreas del currículo [Learning to write/reading to learn: Genre knowledge and pedagogy in the SydneySchool: scaffolding democracy in Literacy Classrooms] (Francisco Lorenzo Bergillos). Buxarrais, M. R., \& Vilafranca, I. (Coords.). Una mirada femenina de la educación moral [A feminine view of moral education] (Eric Ortega González).

351

This is the English version of the research articles and book reviews published originally in the Spanish printed version of issue 273 of the revista española de pedagogía. The full Spanish version of this issue can also be found on the journal's website http://revistadepedagogia.org.

ISSN: 0034-9461 (Print), 2174-0909 (Online)

https://revistadepedagogia.org/

Depósito legal: M. 6.020 - 1958

INDUSTRIA GRÁFICA ANZOS, S.L. Fuenlabrada - Madrid 